\title{
Selective Liposomal Transport Through Blood Brain Barrier Disruption in Ischaemic Stroke Reveals Two Distinct Therapeutic Opportunities
}

\author{
Authors: Zahraa S. Al-Ahmady *1,2 ${ }^{1}$, Dhifaf Jasim ${ }^{1}$, Sabahuddin Syed Ahmad ${ }^{1}$, Raymond \\ Wong ${ }^{3}$, Michael Haley ${ }^{3}$, Graham Coutts ${ }^{3}$, Ingo Schiessl ${ }^{3}$, Stuart M. Allan *3 and Kostas \\ Kostarelos *1
}

\begin{abstract}
Affiliations:
${ }^{1}$ Nanomedicine Lab, Faculty of Biology, Medicine and Health, AV Hill Building, The Univerdsity of Manchester, Manchester M13 9PT, United Kingdom.

${ }^{2}$ Pharmacology Department, School of Science and Technology, Nottingham Trent University, Nottingham, NG11 8NS

${ }^{3}$ Lydia Becker Institute of Immunology and Inflammation, Division of Neuroscience and Experimental Psychology, School of Biological Sciences, Faculty of Biology, Medicine and Health, University of Manchester, Manchester Academic Health Science Centre, AV Hill Building, Manchester, M13 9PT.
\end{abstract}

*To whom correspondence should be addressed: zahraa.al-ahmady@ntu.ac.uk; stuart.allan@manchester.ac.uk; kostas.kostarelos@manchester.ac.uk 


\begin{abstract}
:
The development of effective therapies for stroke continues to face repeated translational failures. Brain endothelial cells form paracellular and transcellular barriers to many blood-borne therapies and the development of efficient delivery strategies is highly warranted. Here, in a mouse model of stroke, we show selective recruitment of clinically used liposomes into the ischaemic brain that correlates with biphasic blood brain barrier (BBB) breakdown. Intravenous administration of liposomes into mice exposed to transient middle cerebral artery occlusion took place at early $(0.5 \mathrm{~h}$ and $4 \mathrm{~h})$ and delayed (24h and $48 \mathrm{~h})$ timepoints, covering different phases of $\mathrm{BBB}$ disruption after stroke. Using a combination of in vivo real-time imaging and histological analysis we show that selective liposomal brain accumulation coincides with biphasic enhancement in transcellular transport followed by a delayed impairment to the paracellular barrier. This process precedes neurological damage in the acute phase and maintains long-term liposomal co-localisation within the neurovascular unit, which could have great potential for neuroprotection. Levels of liposomal uptake by glial cells are similarly selectively enhanced in the ischaemic region late after experimental stroke (2-3 days), highlighting their potential for blocking delayed inflammatory responses or shifting the polarization of microglia/macrophages towards brain repair.
\end{abstract}

These findings demonstrate the capability of liposomes to maximise selective translocation into the brain after stroke and identify two windows for therapeutic manipulation. This emphasizes the benefits of selective drug delivery for efficient tailoring of stroke treatments. 
Stroke is a devastating neurological condition and a leading cause of death and disability worldwide, yet treatment options are extremely limited and thus represent an area of unmet clinical need ${ }^{1,2}$. At present, restoration of blood flow with thrombolysis and/or thrombectomy are the only licensed treatments for ischaemic stroke, however these options can only be administered up to $4.5 \mathrm{~h}$ post-stroke, benefitting only a minority of patients ${ }^{3}$. While reperfusion strategies are effective in opening up occluded cerebral vessels in some patients, there are currently no approved treatments for the myriad of damaging pathological processes that persist in the brain long after the acute stage such as oxidative stress and inflammation ${ }^{4}$.Therefore, targeting these downstream pathophysiological processes could hold great therapeutic potential. However considerable research effort has been invested over the last 30 years into the development of novel neuroprotective treatments, with a lack of success 5 . There are many possible explanations for this translational failure, but insufficient concentrations of drug that reach the intended target area is likely a major factor ${ }^{6}$. Another important problem is the time window between the onset of stroke and treatment initiation which has been frequently wider in clinical trials compared to successful experimental stroke studies ${ }^{4}$. Therefore, developing technologies that can circumvent inefficient brain delivery and/or unfavourable distribution and safety profiles would lend different prospect to already existing therapeutics.

In normal conditions, brain endothelial cells (BECs), through tightly regulated transcellular transport and tight junction (TJ) proteins ${ }^{7}$, are the primary regulators for the entry of blood-borne molecules into the brain. During stroke there is strong evidence from preclinical ${ }^{8-10}$ and clinical $^{11}$, 12 studies that blood brain barrier (BBB) integrity is compromised. Ischaemic conditions affecting the brain tissue alter the rate and the extent of BEC transcellular transport and change the expression levels and localisation of TJ proteins ${ }^{9}$. Moreover, degradation of extracellular matrix by proteolytic enzymes (such as matrix metalloproteinases) ${ }^{13}, 14$, release of inflammatory mediators and infiltration of peripheral blood leukocytes ${ }^{15}$ have all been proposed to contribute to BBB hyperpermeability after ischaemic stroke. As a result, a biphasic increase in BBB hyperpermeability and uncontrolled entry of molecules to the brain occurs. The exact contribution of transcellular and paracellular pathways to BBB hyperpermeability after ischaemic stroke is a matter of controversy ${ }^{16}$. However, the most accepted model of hyperpermeability is characterised by; a) an early phase (occurring a few hours post stroke) of enhanced transcellular transport mediated by increases in endothelial vesicles termed caveolae, followed by b) a delayed phase of hyperpermeability ( 2d post-stroke) in which both enhanced transcellular transport and TJ proteins disassembly contribute to the loss of BBB integrity ${ }^{9}$. This two-phase model is supported by recent evidence in which endothelial TJs were labelled with eGFP, allowing the dynamics of TJ integrity after stroke in mice to be monitored in vivo in real time 9 . Similarly, in a rat model of stroke it was shown that BBB opening to macromolecules (transcellular route) precedes permeability to small ions (paracellular route) ${ }^{17}$. Moreover, recent findings in a comorbid rodent model of ischaemic stroke, reported a significant exacerbation to the BBB disruption when combined with other diseases without necessarily altering the 
underlying sequence of events behind BBB disruption ${ }^{10}$. Consequently, BECs that survive the ischaemic damage, but do not maintain BBB integrity, can in fact worsen the damage to the brain parenchyma and thus accelerate disease progression ${ }^{18}$. On the other hand the disrupted BBB could act as a gate for therapeutic access ${ }^{7}$. This highlights the need for more effective delivery approaches that can selectively and efficiently penetrate areas of BBB hyperpermeability compared to other brain regions where BBB permeability is unaffected.

In the past few decades, nanotechnology-based drug delivery approaches such as liposomes have demonstrated a great potential to improve the pharmacokinetics and biodistribution profile of many small drug molecules. Selective accumulation of liposomes into the disease site, such as a tumour, is mediated by hyperpermeability of endothelial cells and impaired lymphatic drainage compared to other healthy organs. This phenomenon is collectively known as the enhanced permeability and retention (EPR) effect ${ }^{19}$, which is behind the clinical use of many liposomalbased medications ${ }^{20}$. Previous studies have shown that endothelial cell hyperpermeability is mediated by one or more of the following pathways; a) fenestrations in the basement membrane ${ }^{21}$, b) paracellular transport through gaps between endothelial cells ${ }^{21}$, and c) transcellular route mediated by vascular transport (caveolae ${ }^{22}$. These pathways are in great analogy to BECs structural adaptations after ischaemic stroke and, therefore, highlight the possibility that liposomes might be equally effective in the treatment of stroke by providing selective and enhanced drug delivery to the ischaemic brain. In this respect there are a few promising examples of liposomal treatment of ischaemic stroke reported recently ${ }^{23-28}$. However, the focus of those studies is restricted to the acute phase $(<1-3 h)$ after ischaemic reperfusion and they did not fully address longer-term pathological consequences and the practicality of clinical translation. Given that the main aim of neuroprotective treatment of stroke is to expand the therapeutic window compared to thrombolytic therapies, an in depth understanding of the window of BBB opening after stroke is required to fully achieve the potential of liposomes as a drug delivery approach.

The aim of this study was to fully interrogate the validity of utilizing liposomes to maximise drug delivery in stroke. Multiple quantitative (e.g. DiI-Lp intensity, \% of ID of ${ }^{111}$ In-DTPA-Lp) and qualitative techniques (e.g. SPECT/CT, IVIS, confocal imaging and in vivo multiphoton imaging) were employed to establish the time window and mechanism of liposomal accumulation into the brain after experimental stroke in mice. Early and delayed accumulation of liposomes into the brain were evaluated and time points that gave rise to key brain accumulation are highlighted for future therapeutic evaluation. 


\section{Results}

In order to establish the validity of liposomes for selective drug delivery into the ischaemic brain, we have applied in vivo and ex-vivo imaging techniques to study their recruitment into the brain after stroke. A liposomal formulation based on the composition of clinically used liposome (Doxil ${ }^{\circledR}$, HSPC:CHOL:DSPE-PEG 2000 ) was selected, as this has proved to have excellent blood circulation time and very good drug retention capability once inside the body ${ }^{29}$ (For characterisation data please see Figure S1). Selective recruitment of liposomes into the brain was studied in a preclinical model of middle cerebral artery occlusion (MCAo) followed by reperfusion.

\section{Selective liposomal accumulation into the ischaemic brain correlated with biphasic BBB}

breakdown induced by stroke. Liposomal accumulation into the ischaemic brain was first studied shortly after intravenous (I.V) administration of fluorescently-labelled liposomes (DiILp). Each group of mice received a single I.V injection of DiI-Lp. The time points of injections were carefully selected to cover the two-phases of BBB damage after ischaemic stroke as previously reported ${ }^{9}$. IVIS Lumina II imaging (Figure 1A) confirmed the selective accumulation of liposomes into the ischaemic left side of the brain as early as $2 \mathrm{~h}$ after DiI-Lp I.V administration compared to minimum detection from the contralateral side of the brain (right) and healthy mice (naïve mice injected with DiI-Lp). Quantification of the total fluorescent signal of liposomes in the brain indicated that the accumulation of liposomes significantly increased when injected at $0.5 \mathrm{~h}$ or $48 \mathrm{~h}$ after MCAo and reperfusion, by 4.6 -fold at both time points, compared to healthy mice injected with DiI-Lp (Figure 1B). In contrast 2-fold increase in DiI-Lp accumulation observed when injected $4 \mathrm{~h}$ and $24 \mathrm{~h}$ after MCAo and reperfusion. This suggested that liposomal brain accumulation induced by stroke is correlated with the biphasic BBB breakdown in agreement with previous reports. ${ }^{9}, 14,30,31$ Histological analysis of the brain tissue confirmed that the selective liposomal accumulation into the ischaemic area has the same distribution to BBB disruption (Figure 1D), as seen by infiltration of endogenous immunoglobulin (IgG). Selective brain accumulation covered both ipsilateral striatum and cortex when injected 0.5-2.5h and 48-50h after MCAo.

\section{Liposomal brain accumulation in the acute phase post ischaemic stroke preceded neurological} damage. Cerebral accumulation of DiI-Lp injected at the early phase after MCAo and reperfusion (0.5-2.5h \& 4-6h) were observed as early as $2 \mathrm{~h}$ after I.V administration, before the observation of significant histological evidence of neuronal cell death (Figure 1C\&D). Infarct volume measurements were $\sim 5 \mathrm{~mm}^{3}$ for $0.5-2.5 \mathrm{~h}$ and $4-6 \mathrm{~h}$ groups compared to $>30 \mathrm{~mm}^{3}$ observed for 24-26h and 48-50h groups. This early accumulation was associated with rare instances of vessel lumen collapse as seen by H\&E staining (Figure 1D), but in the absence of any active bleeding process. 
Liposomes maintained selective accumulation in the ischaemic area of the brain $24 \mathrm{~h}$ after I.V administration. Selective enhancement of cerebral accumulation of DiI-liposomes given $0.5 \mathrm{~h}$ and $48 \mathrm{~h}$ after reperfusion was still observed in the ischaemic region 24h after their I.V injection. Quantification of DiI-Lp signal in the brain indicated 7.2-fold and 3.9-fold increase compared to healthy mice at $0.5-24.5 \mathrm{~h}$ and $48-72 \mathrm{~h}$, respectively (Figure 2C). DiI-Lp cerebral accumulation covered both ipsilateral cortex and striatum and maintained similar distribution to the areas of BBB damage and infarct as seen with IgG infiltration and Cresyl violet stain respectively (Figure 2D). Liposomal accumulation at the time windows of maximum selective recruitment into the brain $(0.5 \mathrm{~h} \& 48 \mathrm{~h})$ were also studied in real time by SPECT-CT imaging (Figure $3 \mathrm{~A})$ using ${ }^{111} \mathrm{In}$ DTPA-liposomes ( ${ }^{111} \mathrm{In}-\mathrm{Lp}$ ). Characterisation data of ${ }^{111} \mathrm{In}$-Lp and radiolabelling efficiency are explained in Figure S2. During real time SPECT/CT imaging the selective recruitment of liposomes in the brain became more apparent $24 \mathrm{~h}$ after injection since immediately after injection the high level of liposomes in the blood gave rise to equal signal from both sides of the brain (Figure 3B). Quantification of ${ }^{111} \mathrm{In}-\mathrm{Lp}$ accumulation 24h after I.V administration confirmed significant increase in ipsilateral accumulation of ${ }^{111} \mathrm{In}-\mathrm{Lp}$ when injected at $0.5 \mathrm{~h}$ or $48 \mathrm{~h}$ after MCAo (Figure 3E). On the contrary no significant difference in ${ }^{111} \mathrm{In}-\mathrm{Lp}$ levels was detected in the contralateral region (Figure 3F). Measurements of ${ }^{111} \mathrm{In}-\mathrm{Lp}$ in the cerebrospinal fluid (CSF) (Figure 3G) indicated no significant increase in liposomal clearance into the CSF after MCAo, which explained the persistence of liposomes in the ischaemic area even 1d after administration.

\section{Biphasic increase in liposomal brain accumulation after ischaemic stroke connected with enhanced transcellular transport.}

To gain an in depth understanding of the mechanisms of selective liposomal recruitment into the brain after stroke, we investigated the molecular pathways (transcellular vs paracellular) that could be involved. Experimental MCAo model leads to a biphasic increase in transcellular transport across the BBB in the early and delayed phases after ischaemia (Figure 4A). However, it is unclear if enhanced transcellular transport (endothelial caveolae) is connected to the biphasic increase in liposomal brain accumulation after ischaemic stroke. Caveolae are invaginations of plasma membrane that play an important role in the transcellular transport of small molecules such as cholesterol and albumin ${ }^{32}$. Among the caveolins family, caveolin-1 (Cav-1) is known as an important regulator of caveolin mediated transport. The exact contribution of Cav-1 to BBB hyperpermeability is not clear, though recent findings indicate enhanced Cav-1 expression by BECs and increased numbers of transcellular vesicles shortly after ischaemic insult and before TJ disassembly 9, 33, 34. Moreover, Knowland et al confirmed that this effect is in fact biphasic and contributes to both early and late BBB disruption ${ }^{9}$. Consistent with previous reports ${ }^{35,36}$, TEM images of brain tissues in the acute $(\mathrm{MCAo}+2.5 \mathrm{~h})$ and late $(\mathrm{MCAo}+50 \mathrm{~h})$ phases after stroke (Figure 4B) confirmed ultrastructural changes in endothelial cells compared to healthy brains. Increases in caveolae numbers were clearly evident in the cytoplasm of BECs at both time points. Enlargement of the vesicles and 
ultrastructural changes to endothelial cell TJs (protrusions and change in morphology) were also observed at the late but not the early time point after MCAo. These observations were further confirmed by immunofluorescence labelling of endothelial CD31 and Cav-1 markers. We transcardially perfused the animal with PBS under terminal anaesthesia prior to fixing the brain with $4 \%$ PFA. This perfusion ensures removal of any circulating liposomes, meaning that only liposomes taken up by brain endothelial cells (co-localised with CD31) or those extravasated into the brain parenchyma would be imaged (Figure S3). Analysis of the brain tissues $2 \mathrm{~h}$ after intravenous administration of DiI-Lp revealed a biphasic increase in Cav-1 expression from $0.5 \mathrm{~h}(\mathrm{MCAo}+2.5 \mathrm{~h})$ and $48 \mathrm{~h}$ groups $(\mathrm{MCAo}+50 \mathrm{~h})$ at $53 \% \pm 14 \%$ and $28 \% \pm 5.8$, respectively. Areas of enhanced Cav-1 expression co-localised with the areas of DiI-Lp leakage into the ischaemic brain (Figure 4C\&D). Similar observations were detected in the ipsilateral cortex, however to a slightly lesser extent (Figure S4). On the contrary, Cav-1 expression and accumulation of DiI-Lp in the brain were minimum from MCAo groups injected at $4 \mathrm{~h} \& 24 \mathrm{~h}$ post stroke. Similarly, low level of basal Cav1 staining was observed in the healthy brain (Figure 4C and Figure 4SC) and in the contralateral sides (Figure S6 \& Figure S7) which explains limited DiI-Lp accumulations in those cases. Our data agree with Knowland et al observations ${ }^{9}$, where they showed limited basal Cav1 expression in the healthy and contralateral side and a significant increase in Cav1 expression in the ipsilateral side after MCAo.

To confirm the role of caveolae in the uptake of liposomes by brain endothelial cells, we have used dynasore, a pharmacological inhibitor of caveolar transport in hCMEC/D3 brain endothelial cells in vitro. Consistent with our in vivo data, we observed a significant reduction $(\sim 80 \%)$ in DiI-Lp uptake by hCMEC/D3 cells in the presence of dynasore (Figure 3E). In comparison, no significant reduction in DiI-Lp cellular uptake was observed with chlorpromazine, an inhibitor of clathrin-mediated transport. Overall, our data suggested that caveolae is the main pathway behind liposomes mediated transcytosis through brain endothelial cells.

Evaluation of DiI-Lp localisation 24h following I.V at $0.5 \mathrm{~h}$ and $48 \mathrm{~h}$ to MCAo mice (Figure 3F) indicated that substantial numbers of liposomes maintain their co-localisation in the neurovascular unit (NVU) in areas of enhanced Cav-1 expression (Figure 4E \& Figure S5).

\section{Delayed liposomal brain accumulation after ischaemic stroke co-localised with regions of impaired paracellular barrier}

In addition to enhanced transcellular transport, compromised paracellular barrier by virtue of $\mathrm{TJ}$ proteins disassembly leads to BBB disruption after ischaemic stroke. Up to $30 \%$ of TJ strands were reported to be open 48-58h after MCAo and the opening of these gaps increased progressively after stroke, reaching gaps of 0.2-1.2 $\mu \mathrm{m}^{9}$. Since the size of TJ opening is far bigger than the hydrodynamic diameter of liposomes tested in this study $(120-130 \mathrm{~nm})$ it is 
feasible that the delayed phase of liposomal accumulation is facilitated by the paracellular route. To test this, mice were implanted with a cranial window underwent MCAo surgery and received DiI-Lp intravenously at $0.5 \mathrm{~h}$ or $48 \mathrm{~h}$ after MCAo. Together with DiI-Lp injections, mice also received I.V injections of fluorescent tracers for paracellular pathway (dextran 3k) and transcellular pathway (albumin-Alexa488). Previous studies have demonstrated that loss of TJ integrity results in increased vascular permeability to markers ranging from 0.5 to $300 \mathrm{kDa}$. Therefore, we have selected dextran $3 \mathrm{kDa}$ for this study as it is within that molecular weight range and at the same time being not a very large molecule can allow the detection of even slight changes in TJ integrity ${ }^{37}$. This is important to prove that $\mathrm{TJ}$ integrity is maintained in the early phase after MCAo as selecting a dextran molecule with a larger molecular weight might give a negative indication of the absence of paracellular transport due to a size exclusion effect ${ }^{38}$. To examine the dynamic accumulation of liposomes into the ischaemic brain and correlate that with structural abnormalities of BECs, we subjected MCAo mice to in vivo multiphoton imaging recording at $30 \mathrm{~min}$ and $2 \mathrm{~h}$ after DiI-Lp and tracers injections. These time points were selected to match our previous data sets and offered the advantage of having both time points in the same animal.

Dextran is normally excluded from the brain parenchyma by an intact $\mathrm{TJ}$ as can be seen from multiphoton images (Figure 5B), however, its extravasation from the blood vessels increased by 2-fold in the late phase after ischaemic stroke progression (Figure 5C). This extravasation became apparent at 48-50h after MCAo and reperfusion, correlating with the TJ disassembly. In contrast, albumin uptake by BECs was reported to be through caveolae mediated endocytosis ${ }^{39}$, thus its accumulation into the brain increased by 5.9 -fold in the acute phase $(0.5-2.5 \mathrm{~h})$ and by 3 fold in late phase (48-50h) after MCAo (Figure 5B\&C). These observations corroborated our previous findings from TEM and IHC analysis, suggesting that BBB permeability in the early phase is mediated mainly by enhanced transcytosis. This is also in agreement with other recent studies of BBB permeability in MCAo model ${ }^{9,10}$. The observations of real time multiphoton imaging were further validated ex vivo using IVIS of the brain which again demonstrated biphasic enhancement in albumin and DiI-Lp after stroke, compared to only delayed accumulation of dextran at 48-50h after MCAo (Figure 5D\&E).

Overall, our data suggest that biphasic upregulation of transcellular pathway followed by delayed TJ disassembly are the drive for the selective liposomal accumulation into the ischaemic side of the brain (Figure 5B\&C). Although Cav-1 is known to be essential for transcellular transport of albumin through BECs, recent observations showed that albumin uptake could still be enhanced in Cav-1 deficient mice, though to a lesser extent, which imply the contribution of other Cav1-independent pathways ${ }^{9}$. Moreover, previous studies have also reported that the transcytosis rate of endothelial cells is also affected by other factors such as; changes in Cav-1 phosphorylation ${ }^{40}$, loss of Mfsd2a (major facilitator superfamily domain containing 2a) expression (suppressor of transcytosis) ${ }^{41}$ or upregulation of other transcytosis proteins such as 
Cavin-1, Cavin- ${ }^{32}$. Therefore, it would be important to investigate the correlation of those factors to enhanced liposomal accumulation in the brain in the future.

Selective targeting of ischaemic brain with liposomes offers time-dependent tailoring of stroke treatment. Immunobiological analysis described above indicated that liposomes administered in the acute $(0.5 \mathrm{~h})$ and late $(48 \mathrm{~h})$ phases after stroke co-localised with the NVU. Since the NVU plays a critical role in the progression of ischaemic damage after stroke, selective targeting of the NVU with liposomes, particularly in the acute phase, could hold great potential as a therapeutic strategy by limiting the deleterious effects of BBB disruption after stroke.

In addition to the NVU, co-localization of the liposomes with other cellular compartments of the brain parenchyma was tested. Immunostaining for ionised calcium binding adaptor molecule 1 (Iba-1), which is exclusively expressed by macrophages and microglia and glial fibrillary acifdic protein (GFAP), expressed by astrocytes, was performed (Figure 6). The uptake of fluorescently labelled liposomes (DiI-Lp) by microglia and astrocytes was investigated with confocal microscopy by measuring the co-localisation of DiI-Lp signal with Iba-1 and GFAP +ve areas. Confocal images showed very limited liposomal uptake by astrocytes at both time points (Figure 6A). Where as a clear uptake was observed by microglia only at the 48-50h after MCAo (Figure 6B\&C) which was not observed 0.5-2.5h after MCAo (Figure 6A). These observations were confirmed by quantification of the percentage of DiI-Lp uptake by Iba1+ cells and GFAP+ cells which was performed 24h after I.V administration of DiI-Lp. We found that a significant fraction (40-50\%) of DiI-Lp co-localised with Iba1+ cells when administered 2 days after MCAo compared to <3-4\% when injected in the acute phase, at $0.5 \mathrm{~h}$ after MCAo (Figure $6 \mathrm{~F})$. Resting microglia dynamically monitor the brain microenvironment ${ }^{42}$, and become activated when sensing damaging signals, changing morphology from long, ramified protrusions

to shorter, hyper-ramified branches ${ }^{43}$. Our results demonstrate DiI-Lp uptake by both resting and activated microglia, though it is enhanced in the latter (Figure 6G). In contrast, less uptake (< $5 \%$ ) of DiI-Lp by Iba-1 +ve cells was observed in the ipsilateral cortex at both time point (Figure S8). Minimum uptake of DiI-Lp by astrocytes was observed at both times tested both in the ipsilateral striatum (Figure 6H) and cortex (Figure S8). No specific uptake of DiI-Lp by neurons was detected at the time points tested, while occasional co-localisation with MBP+ cells (oligodendrocytes) was observed (Figure S9 \& Figure S10).

\section{Discussion}

Stroke-induced BBB disruption results in a biphasic enhanced entry of blood borne molecules to the brain. Despite the obvious benefits this can offer to enhance drug delivery to the ischaemic brain, this area has been largely overlooked. Understanding the link between the response of BECs in vivo to ischaemic injury and selective drug delivery to the brain is crucial to the development of timely therapies that may halt disease progression. 
Recent studies using nanoparticles-based delivery systems demonstrated the possibility to selectively target the lesion area after experimental stroke. This effect was only demonstrated when those nanoparticles injected I.V just before reperfusion or 1-3 h afterward ${ }^{44,45}$. Although these studies suggested that the increase in the permeability of the BECs is the drive for this selective accumulation, no direct correlation to BBB damage was reported. Moreover, the exact mechanisms by which the selective brain localization happens are largely unknown and require in depth studies. Therefore, in this study we systematically interrogated the benefit of BBB damage to enhance drug delivery by analysing the brain accumulation and localisation of I.V liposomes injected at different time points after experimental stroke to cover the biphasic increase in BBB permeability. The most acceptable model of BBB response to ischemia involves a stepwise activation of two distinctive pathways, starting with enhanced BECs transcellular transport early after reperfusion followed by a second delayed phase ( $48 \mathrm{~h}$ post stroke) of enhanced transcellular transport and major disassembly to TJ protein complexes ${ }^{9,46}$.Here we demonstrate that biphasic BBB hyperpermeability after stroke correlates with selective enhancement of liposomes accumulation into the ischaemic brain. More specifically we identified two distinct windows for maximum localisation of liposomes into the brain; an early phase $(0.5 \mathrm{~h}$ after stroke) that agrees with previous studies, and a delayed phase ( $48 \mathrm{~h}$ after stroke) which has not been reported before. These observations were based on in vivo (SPECT/CT and multiphoton imaging) and ex-vivo (IVIS) imaging techniques and were further confirmed by histological analysis. We have demonstrated that enhanced transcellular transport is the main mediator for increase liposomal brain accumulation in the early phase $(0.5 \mathrm{~h})$. Whereas the reduction in the level of these vesicles in the time between $4 \mathrm{~h}$ and $24 \mathrm{~h}$ after stroke is behind the minimal accumulation of liposomes observed at those time points. This agrees with previous studies that reported limited liposomal translocation or therapeutic effect from delivery systems administered around those times ${ }^{44,45}$. Our data also reveals a second window for liposomal entry into the brain $(\sim 48 \mathrm{~h}$ after stroke) in which both transcellular and paracellular pathways contribute to the selective localisation of liposomes.

The reasons behind the initial increase in transcellular transport through BECs after ischemia are not well defined yet. One possible explanation, correlates with the early migration of pericytes away from the BECs that is triggered after basement membrane dissolution ${ }^{47,48}$. Pericytes are known to secrete inhibitory signals that reduce the rate of transcellular transport through BECs. Thus, the loss of those inhibitory signals can lead to the initial rise in transcellular transport ${ }^{49,50}$. Caveolae are the intermediaries of this transcellular pathway and contain receptors to molecules that must cross the $\mathrm{BBB}{ }^{51}$, However, it is unclear if this increase has any benefit the to NVU at this stage after stroke ${ }^{9}$. The late increase in BBB hyperpermeability is more linked to TJ disassembly triggered by matrix metalloproteinases (MMPs), secreted by inflammatory cells, and angiogenic growth factors such as vascular endothelial growth factor and $\&$ nitric oxide synthase ${ }^{9,15}$. This comes in parallel with a second phase of increase in BECs transcytosis ${ }^{9}$. Although previous studies reported reduced level of TJ proteins (Claudin5, 
Occludin, and Zona Occludens-1) in the first few hours after stroke ${ }^{31}$, ultrastructural analysis of TJ during the evolution of ischaemic stroke are not persuasive that this reduction is responsible for the early BBB breakdown ${ }^{34,52}$. Based on the studies mentioned above, it is suggested that active disassembly of TJ proteins occur in the late phase of reperfusion, when angiogenesis of CNS vessels begins ${ }^{53}$. This is also in agreement with our data that demonstrated TJ modification only $48 \mathrm{~h}$ after MCAo. This was confirmed with TEM imaging of brain sections and multiphoton and IVIS imaging of dextran $3 \mathrm{k}$ extravasation into the brain.

Identification of the exact therapeutic benefit of this selective brain delivery approach needs substantially more experimental work. Here we provide a clear evidence that selective accumulation of liposomes into the ischemic hemisphere precedes neuronal damage which make them ideal for neuroprotection. Few neuroprotective drugs have been tested in liposomal formulation injected at reperfusion or shortly after such as erythropoietin ${ }^{26}$, tacrolimus ${ }^{44}$ and CDP-choline ${ }^{28}$. When compared to free drugs, promising therapeutic effects were reported including reduced cellular apoptosis, brain infarct volume and improved motor function.

Beside protecting the brain tissue, NVU itself can be a therapeutic target. It is well known that disruption of the BBB markedly influences the pathogenesis of stroke. Moreover, both systemic and cerebrovascular inflammation contribute to further BBB disruption and can alter stroke prognosis. This effect is both neutrophil and MMPs dependent ${ }^{54}$. Neutrophils are generally considered the first responders to ischaemic stroke as they infiltrate the brain within the first few hours after the ischaemic insult. Neutrophils have a key role in BBB disruption as they are the source of various proteolytic enzymes in particular MMPs. Consistent with that, recent studies indicated that active MMP2/9 observed in the brain after the initial disruption of BBB permeability, approximately $3 \mathrm{~h}$ after ischemia ${ }^{55}$, and progressively increased afterwards ${ }^{56}$. In vitro and in vivo studies suggested that inhibition of MMP2/9 minimised BECs permeability and transiently reduced the infarct volume. Our immunobiological analysis indicated substantial colocalisation of liposomes with NVU both in the early and delayed phases after stroke which was maintained even $24 \mathrm{~h}$ after administration. The therapeutic potential of this has not been tested before. However due to the great influence of NVU on the progression of ischaemic damage after stroke, selective targeting of the NVU with liposomes, could be used to influence neutrophil infiltration and/or for MMPs inhibition in order to mitigate the deleterious effects of BBB disruption after stroke.

Cerebral inflammation is also a key factor in neuronal damage after acute ischaemic injury ${ }^{57}$. This is mainly mediated by the secretion of pro-inflammatory mediators by classically activated M1 microglia and macrophages such as interleukin-1 family which are triggered in response to sterile inflammation ${ }^{58}$. However, recent studies highlighted the dual role of microglia/macrophages in brain disorders. In fact, alternatively activated M2 phenotype are the source of protective and neurotrophic factors that reserve brain function and promote brain 
functional recovery ${ }^{59}$. Therefore, blocking the inflammatory responses after stroke ${ }^{60-62}$ or shifting microglia/macrophage polarisation towards brain repair ${ }^{63}$ is another area of therapeutic potential. The present study is the first to identify the intriguing selective uptake of liposomes by microglia in the late phase after stroke and its correlation with microglia activation stage. Therefore, it will be essential to investigate the potential of liposomes to tip the central inflammatory responses after stroke towards brain repair. This would allow cell type specific intervention to be tested in timely manner after stroke.

The therapeutic targets described above are driven by the natural tendency of selective liposomal accumulation into the brain post stroke, however, active targeting of specific cell population in the brain is another area worth of investigation. This concept can be highly relevant for delivering neuroprotective drugs to neurons early on after stroke, as liposomal accumulation in the brain was evident prior to neuronal cell loss. The approach we are describing could also be translated to other types of nanocarrier (e.g. polymeric nanoparticles, micelles, etc) that could utilise both delivery windows to translocate into the brain after stroke. However, a key advantage liposomes offer compared to other drug delivery approaches, is their ability to encapsulate hydrophilic and hydrophobic molecules. This makes them very attractive for stroke therapy, since the choice of encapsulated therapeutic molecules can be easily tailored to match the therapeutic target in a timely manner.

Since treatment with therapeutic agents in the early phase after ischemia/reperfusion is necessary to preserve brain function, in future studies it is important to investigate the effect of this early intervention on BBB permeability at later time points (e.g. 48h) after stroke since this could be changed by the intervention. This is especially important if a dual targeted approach is utilised to deliver therapeutic molecules at both early and delayed phases after stroke. Another important factor to consider is the effect of stroke severity (MCAo occlusion time) on the timing and mechanism of liposomal brain accumulation into the brain after stroke.

\section{Limitations of the results}

In this study we have established the potential of utilizing liposomes to achieve selective enhanced translation in ischaemic brain after experimental stroke. We have identified twowindows for maximum accumulation into the brain with two distinct therapeutic targets. What we haven't showed in this study is the translation of this enhanced accumulation into improved therapeutic activity or functional recovery by encapsulating therapeutic molecules inside the liposomes. However, this study lays the basis for critical design of future therapeutic studies to prove the potential liposomes offer to accelerate the clinical translation of stroke treatments. 


\section{Conclusions}

We propose liposomal drug delivery to enhance the translocation of therapeutic molecules into the brain by taking advantage of BBB disruption induced by ischaemic stroke. Liposomal transport through the BBB deficits in experimental stroke is mediated by a stepwise impairment of transcellular followed by paracellular barriers. Our data revealed two windows for selective stroke treatment including; a) targeting the NVU in the acute phase to preserve the brain function and minimise the deleterious consequences of stroke, and b) targeting inflammatory cells in the ischaemic brain to shift their polarization towards brain repair. Future studies to test the therapeutic potential of liposomes in ischaemic stroke are warranted to prove their potential utility in accelerating the clinical translation of stroke treatments. 


\section{Materials and Methods \\ Materials.}

Hydrogenated soy phosphatidylcholine (HSPC) and 2-distearoyl-sn-glycero-3phosphoethanolamine- $N$-[methoxy(polyethylene glycol)-2000] (DSPE-PEG 2000 ) were kind gifts from Lipoid GmbH (Ludwigshafen, Germany).18:0 PE-DTPA 1,2-distearoyl-sn-glycero-3phosphoethanolamine-N-diethylenetriaminepentaacetic acid (ammonium salt) was purchased from Avanti Polar Lipids (USA). Chloroform and methanol were purchased from Fisher Scientific. Phosphate buffer saline, cholesterol, paraformaldehydes were purchased from Sigma. 1,1'-Dioctadecyl-3,3,3',3'-Tetramethylindocarbocyanine Perchlorate (DiI) was purchased from Invitrogen Detection Technologies. Polycarbonate extrusion filters (Whatman) 800nm, 200nm, and $100 \mathrm{~nm}$ were form VWR, UK. PD-10 desalting columns were bought from GE-Healthcare Life Sciences.

\section{Mice and diets}

C57BL/6 male mice (11-12-week-old, weighing 25-30 g; Envigo, UK) were housed in groups of 4-5. All mice were given free access to diet and water and were housed at a constant ambient temperature of $21 \pm 2^{\circ} \mathrm{C}$ and humidity of $40-50 \%$, on a 12-h light, 12-h dark cycle. All experimental procedures using animals were carried out according to the United Kingdom Animals (Scientific Procedures) Act, 1986 and approved by the Home Office and the local Animal Ethical Review Group, University of Manchester and reported in compliance with the ARRIVE guidelines.

\section{Studies design and exclusion criteria}

Calculations of sample sizes were based on power analysis of data from pilot studies and previous experiments. For DiI-Lp accumulation in the brain, we have estimated a mean value of 4.0 $\mathrm{E}^{-5}$ for healthy mice and a mean value of $4.3 \mathrm{E}^{-4}$ for MCAo mice with a SD of $1.05 \mathrm{E}^{-4}$. Assuming a significance level of $\leq 0.05$ and a power of $80 \%$, the estimated sample size is $n=3-4$ for detection of $60-70 \%$ difference. For the detection of ${ }^{111} \mathrm{In}-\mathrm{Lp}$ ID in the brain, the mean value for healthy mice is $0.04 \%$ of ID and MCAo mice is $0.24 \%$ with STD of $0.05 \%$ which gives a sample size of $n=3-4$ mice to detect a deference of $50-60 \%$.

The data were excluded from the analysis in the following conditions; 1) lack of sustained reduction in cerebral blood flow and 2) if signs of subarachnoid haemorrhage or seizure were observed. All experimental procedures using animals were carried out according to the United Kingdom Animals (Scientific Procedures) Act, 1986 and approved by the Home Office and the local Animal Ethical Review Group, University of Manchester and reported in compliance with the ARRIVE guidelines.

\section{Induction of focal cerebral ischaemia}

Focal ischaemic stroke was induced by transient middle cerebral artery occlusion (MCAo) as previously described ${ }^{10,64}$. Briefly, under $2 \%$ isoflurane anaesthesia (in a mixture of $30 \%$ oxygen and $70 \%$ nitrous oxide), the carotid arteries were exposed and a 6-0 silicon rubber-coated

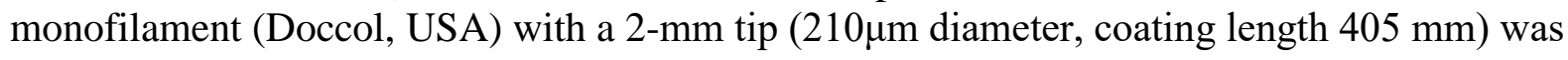
inserted into the left common carotid artery and advanced along the left internal carotid artery 
$10 \mathrm{~mm}$ after the left carotid bifurcation. Cerebral blood flow was monitored in all mice by laserDoppler (Moor Instruments, UK) and MCAo was confirmed by a drop in cerebral blood flow of at least $40-50 \%$ of baseline. If this drop in blood flow was not attained, animals were excluded from the analysis. After 20min occlusion, reperfusion was achieved by withdrawing the filament and the wound was sutured. During surgery, core body temperature was monitored using a rectal probe and maintained at $37 \pm 0.5^{\circ} \mathrm{C}$, using a homoeothermic blanket. Before recovery all mice were given saline $(0.5 \mathrm{ml}$, S.C) and buprenorphine $(0.05 \mathrm{mg} / \mathrm{kg} \mathrm{S.C})$. After surgery, mice were weighed every day and assessed for their general well-being. Body weight data were presented as a \% weight change compared with body weight on the day of surgery. Assessment of cerebral ischemia was performed using the a 28 -point neurological scoring system ${ }^{65}$. Foot fault test was also performed to confirm ischaemic stroke model as previously described ${ }^{64}$. At the end time point of each group, MCAo mice were placed on an elevated grid surface with grid openings of $2.5 \mathrm{~cm}^{2}$. During locomotion on the grid, the number of foot slips of both the ipsilateral and contralateral limbs was recorded. Ipsilateral refers the ischaemic side of the body (left) and contralateral limbs are those on the opposite side (right). Tests repeated for three times for each mouse and each trial lasted for $1 \mathrm{~min}$. An interval of at least $1 \mathrm{~min}$ was kept between each trial. The total number of errors of each side is recorded and expressed as \% of contralateral foot slips.

\section{Preparation of DiI-labelled liposomes (DiI-Lp)}

DiI-labelled liposomes composed of HSPC:Chol:DSPE-PEG 2000 56.3:38.2:5.5 mol/mol \% were prepared by thin film hydration method followed by extrusion. Briefly lipids dissolved in chloroform: methanol mixture (4:1) were mixed in round bottom flask and $5 \mathrm{~mol} \%$ of DiI in ethanol $(1 \mathrm{mg} / \mathrm{ml})$ was added to the lipid mixture. Organic solvents were then evaporated to produce the lipid film ${ }^{66-68}$. Lipid films were kept protected from light and hydration was performed with HBS (20mM HEPES, $150 \mathrm{mM} \mathrm{NaCl}, \mathrm{pH} 7.4$ ) to a final lipid concentration of $12.5 \mathrm{mM}$. To produce small unilamellar liposomes, the size was reduced by extrusion though $800 \mathrm{~nm}$ and $200 \mathrm{~nm}$ polycarbonate filters 5 times each then $20-40$ times through $100 \mathrm{~nm}$ membranes using a mini-Extruder (Avanti Polar Lipids, Alabaster, AL).

\section{Preparation and characterisation of ${ }^{111}$ In-labelled liposomes ( $\left.{ }^{111} \mathbf{I n}-\mathbf{L p}\right)$}

To quantify and study the accumulation of liposomes into the brain in real-time SPECT/CT imaging and gamma counting of the liposomes were performed after radiolabelling with radioactive indium $\left({ }^{111} \mathrm{In}\right.$ ). Briefly, $25 \mathrm{mM}$ (total lipid concentration) of HSPC:Chol:DSPEPEG $_{2000}:$ PE-DTPA 56.3:38.2:5.5:1 $\mathrm{mol} / \mathrm{mol} \%$ liposomes were prepared as described above using thin film hydration method. Hydration of the lipid film was done with freshly prepared ammonium acetate buffer $(0.095 \mathrm{M}, \mathrm{pH} 5.5)$ at $60^{\circ} \mathrm{C}$ followed by extrusion to reduce the size of the liposomes. Subsequently liposomes were radiolabelled by $1 \mathrm{~h}$ incubation with radioactive ${ }^{111} \mathrm{InCl}_{3}(11 \mathrm{MBq} / 2.5 \mu \mathrm{mol}$ lipids) in $2.0 \mathrm{M}$ ammonium acetate $\mathrm{pH}$ 5.5. Incubation carried out at room temperature with continuous vortexing every 5 minutes. At the end of incubation $0.1 \mathrm{M}$ EDTA ( $1 / 20$ of the total volume) was added to chelate any free ${ }^{111} \mathrm{In}$. To determine the Radiolabelling efficiency, any unbound ${ }^{111}$ In and ${ }^{111}$ In-EDTA were removed with PD-10 column pre-equilibrated with HBS pH 7.4. Aliquots of each final product were diluted five folds in PBS and then $1 \mu \mathrm{l}$ was spotted on silica gel impregnated glass fibre sheets (PALL Life Sciences, UK). The strips were developed with a mobile phase of 50mM EDTA in 0.1M ammonium acetate and allowed to dry before analysis. This was then developed and the autoradioactivity quantitatively 
counted using a Cyclone phosphor detector (Packard Biosciences, UK). The immobile spot on the TLC strips indicated the percentage of radiolabelled ${ }^{111} \mathrm{In}-\mathrm{Lp}$, while free ${ }^{111} \mathrm{In}$ was detected as the mobile spots near the solvent front. Very minimum free ${ }^{111}$ In was detected to yield radiolabelling efficiency of $>85 \%$ as displayed in Figure S2. The radiolabelling stabilities of the final product of ${ }^{111}$ In-Lp were studied after five times dilution in both 50\% serum or PBS and then incubated at $37^{\circ} \mathrm{C}$ up to $48 \mathrm{~h}$. At different time-points $(0,1$ and $24 \mathrm{~h}), 1 \mu \mathrm{l}$ of the aliquots was spotted on silica gel impregnated glass fibre sheets and then developed and quantified as described above. No significant release of ${ }^{111} \mathrm{InCl}_{3}$ was detected after incubation with PBS and minimum free ${ }^{111} \mathrm{InCl}_{3}$ was detected after incubation in $50 \%$ for 2 days (Figure S2).

\section{Liposomes characterization}

Liposome size and surface charge were measured using Zetasizer Nano ZS (Malvern, Instruments, UK). Samples were diluted 100 times with purified distilled water before measurements. Triplicates measurements were recorded, and the data expressed as average \pm S.D. Fluorescen intensity of DiI-Lp was recorded using Carry Eclipse fluorescence spectrophotometer, (Agilent technology). Samples were first diluted 200 times in HBS and recorded at $518 \mathrm{~nm} / 565 \mathrm{~nm}$ excitation/emission wavelengths (slit 5/10).

\section{Optical imaging of Lp-DiI accumulation into the brain}

Ex vivo optical imaging was used to study the accumulation of DiI-Lp into the brain by detecting the optical fluorescence signal of the liposomes. IVIS imaging was performed shortly after cardiac perfusion with iced-cold $0.9 \%$ saline followed by $4 \%$ PFA in order to remove any DiI-Lp that are still circulating in the blood. Brain tissues were extracted either $2 \mathrm{~h}$ or $24 \mathrm{~h}$ after I.V administration of DiI-Lp into MCAo mice (at 0.5h, 4h, 24h and 48h after 20min MCAo and reperfusion) and healthy mice. Imaging were performed with IVIS Lumina II imaging system (Caliper Life Sciences Corp., Alameda, CA) at 535nm/DsRed excitation and emission filters with $0.5 \mathrm{sec}$ exposure. DiI-Lp total fluorescence intensity in the brain was quantified by drawing a region of interest (ROI) that covers the whole brain and expressed as total efficiency. We would like to emphasise that this method offers 'semi-quantitative' estimations of fluorescence intensity of the liposomes in the brain and the absolute quantification of the liposomes in the brain as \% of I.D was measured in a separate experiment by gamma counting of ${ }^{111}$ In-liposomes (as explained below).

\section{Single photon emission computed tomography (SPECT/CT)}

Mice were subjected to anaesthesia via the inhalation of $2.5 \%$ isoflurane in a mixture of $30 \%$ oxygen and $70 \%$ nitrous oxide. Each animal was then intravenously injected with $200 \mathrm{ul}$ of the radioactive ${ }^{111} \mathrm{In}-\mathrm{Lp}(8-9 \mathrm{MBq})$. At different time points after injection $(\mathrm{t}=0-1 \mathrm{~h}, \& 24 \mathrm{~h})$ SPECT/CT imaging was carried out using a Nano-Scan ${ }^{\circledR}$ SPECT/CT scanner (Mediso, Hungary). SPECT images were obtained in 20 projections over 40-60 min using a 4-head scanner with $1.4 \mathrm{~mm}$ pinhole collimators. CT scans were taken at the end of each SPECT acquisition using a semi-circular method with full scan, 480 projections, maximum FOV, $35 \mathrm{kV}$ energy, $300 \mathrm{~ms}$ exposure time and 1-4 binning. Acquisitions were done using the Nucline v2.01 (Build 020.0000) software (Mediso, Hungary), while reconstruction of all images and fusion of SPECT with CT images was performed using the Interview ${ }^{\mathrm{TM}}$ FUSION bulletin software (Mediso, Hungary). The images were further analysed using VivoQuant 3.0 software (Boston, 
US) where the SPECT images with scale bars in MBq were corrected for decay and for the slight differences in radioactivity in the injected doses between animals.

For a quantitative assessment of ${ }^{111}$ In-Lp in the brain, a cut and count method was used. Mice were anaesthetized by isofluorane inhalation and each mouse was injected via the tail vein with $200 \mu$ containing ${ }^{111}$ In-Lp labelled with approximately 8-9 MBq. 24h after injection, mice were perfused with iced cold saline $(0.9 \%)$ followed by PFA (4\%) to remove any ${ }^{111} \mathrm{In}-\mathrm{Lp}$ from the blood before brain tissues were collected. Each sample was weighted and counted on a gamma Counter (Perkin Elmer, USA), together with a dilution of the injected dose with dead time limit below $60 \%$. The results were represented as the percentage of the injected dose $(\% \mathrm{ID} / \mathrm{gm}$ tissue \pm SEM), $\mathrm{n}=4-5$ mice per group.

\section{Cranial window implantation and multi-photon imaging}

Cranial windows were implanted following the protocol published by Goldey et. al $2014{ }^{69}$ Animals were anesthetized with $2.5 \%$ isoflurane in $100 \%$ room air. After an injection of Metacam (50 $\mu 1 \mathrm{SC}$ in 1:10 water) and Dexafort (30 $\mu 1 \mathrm{IM})$ the scalp was removed over the stroked hemisphere. Then a metal head plate was mounted (Narishige CP-2, Japan) to allow stereotaxic fixation under the two-photon microscope (Leica SP8 MP, UK HC FLUOTAR L 25x 0.95 WATER dipping lens). A circular piece of bone with a diameter of $3 \mathrm{~mm}$ and centred around the stereotaxic coordinates $2.8-3.35 \mathrm{~mm}$ left, $0.85-1.7 \mathrm{~mm}$ posterior relative to bregma. This region of interest exposes the somatosensory area of the cortex (specifically the barrel cortex) that is most affected by the MCAo. Once the skull was removed a circular cover slip (Warner Instruments, USA) was glued in its place using dental cement (Sun Dental, Japan). After the surgery animals were housed individually and allowed to recover for at least one week and monitored for normal behaviour like nest building and grooming.

In vivo multiphoton imaging was carried out under 1.5\% isoflurane anaesthesia in a 50:50 mix of oxygen and nitrous oxide at 0.5 hours and 48 hours after MCAo. Once anaesthesia was induced DiI-Lp $(100 \mu 1)$, cascade blue dextran 3k $(50 \mu 1)$ and Alexa-488 albumin $(50 \mu 1)$ were injected via the tail vein. For the imaging itself we selected a stroked area of the brain that displayed different size vessels as well as large areas of parenchyma. 3D stacks were recorded up to a depth of $150 \mu \mathrm{m}$ and $\mathrm{z}$ projection images calculated from these with ImageJ.

\section{In vitro cellular uptake studies of DiI-Lp by hCMEC/D3 cells}

In vitro cellular uptake study was performed with hCMEC/D3 cells, an extensively characterised model of brain endothelial phenotype and function, in order to get better insight on the mechanism of liposomal uptake. First, hCMEC/D3 cells were seeded in a 24 well plate at 10,000 cells / well and allowed to adhere for $48 \mathrm{~h}$ before performing the cellular uptake study. To determine the role of caveolae mediated transcytosis in the uptake of liposomes, we incubated hCMEC.D3 cells with $0.5 \mathrm{mM}$ of DiI-Lp for $17 \mathrm{~h}$ in the presence and absence of dynasore $(25 \mu \mathrm{g} /$ $\mathrm{ml}$ ) that inhibits dynamin dependent internalisation of caveolae ${ }^{70,71}$. As a control, we also tested DiI-Lp cellular uptake after inhibition of clathrin-mediated endocytosis using chlorpromazine $(10 \mu \mathrm{g} / \mathrm{ml})^{72}$. At the end of the incubation time, cells were washed twice with Optimum media and imaged using IncuCyte at Ex $518 \mathrm{~nm}$ and Em $565 \mathrm{~nm}$ to quantify cellular uptake. 


\section{Tissue processing}

Tissue processing was carried out $2 \mathrm{~h}$ or $24 \mathrm{~h}$ after liposomes injections. Mice were terminally anaesthetised with isoflurane and perfused transcardially with iced-cold $0.9 \%$ saline and then fixed with $4 \%$ paraformaldehyde (PFA) in $0.1 \mathrm{M}$ phosphate buffer saline (PBS). Subsequently, brain samples were removed and post-fixed (in 4\% PFA) overnight, cryoprotected in 30\% sucrose for $1-2 \mathrm{~d}$ and snap frozen in isopentane on dry ice. Coronal brain sections $(30 \mu \mathrm{m})$ were cut on a freezing sledge microtome (Bright 8000-001, Bright Instrument Co Ltd, UK) and stored in cryoprotectant (30\% ethylene glycol, $20 \%$ glycerol in $0.2 \mathrm{M}$ phosphate buffer) at $-20^{\circ} \mathrm{C}$ until further processing.

For electron microscopy (EM), mice were perfused with $0.9 \%$ saline for $1 \mathrm{~min}$ at $10 \mathrm{ml} / \mathrm{min}$ followed by fixative (4\% PFA and $2.5 \%$ glutaraldehyde in $0.2 \mathrm{M} \mathrm{HEPES}$ ) for $3 \mathrm{~min}$. After that brains were removed and post-fixed overnight. Slices were cut at $1 \mathrm{~mm}$ thin then selected areas of striatum and cortex at $0.14 \mathrm{~mm}$ from the bregma were dissected out and processed for EM as previously described ${ }^{10}$. Briefly, after primary fixation tissues were fixed for $1 \mathrm{~h}$ with $1.5 \%$ potassium ferrocyanide and $2 \%$ osmium tetroxide (weight/vol) in $0.1 \mathrm{M}$ cacodylate buffer and $1 \%$ uranyl acetate at $4^{\circ} \mathrm{C}$ overnight. The next day, samples were dehydrated with serial dilutions of alcohol and embedded in TAAB Low viscosity epoxy resin (TAAB, UK). Ultrathin sections (70nm) were cut from resin-embedded samples on an ultramicrotome (Reichert Ultracut), mounted on Formvar-coated grids and viewed on a FEI Tecnai 12 Biotwin Transmission Electron Microscope. Images were acquired with Gatan Orius SC1000 CCD camera.

\section{Assessment of ischaemic damage}

Brain sections were stained with cresyl violet and the infarct volume was calculated by measuring the areas of neuronal loss at eight defined coronal levels as previously described ${ }^{73,74}$. On each section the area of damage was measured using Image J (NIH, Bethesda, MD, USA), adjusted for oedema and the volume of damage calculated by integration of areas of damage with the distance between coronal levels using GraphPad Prism 7, Software. The volume of damage was expressed as the total amount of ischaemic damage. For the assessment of haemorrhagic transformation, haematoxylin and eosin $(\mathrm{H} \& \mathrm{E})$ staining was performed. The area of red blood cells was measured in the same way as the infract volume was calculated and compared between the groups.

\section{Assessment of BBB permeability to IgG}

To assess BBB permeability, endogenous IgG accumulation into the brain was visualised by

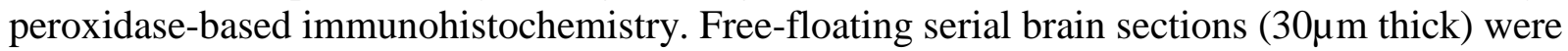
washed 3 times with PBS and endogenous peroxidase activity and non-specific staining were blocked by 10 min incubation in $0.3 \% \mathrm{H}_{2} \mathrm{O}_{2}$ followed by washing ( 3 times in PBS, 10min each). After that $1 \mathrm{~h}$ blocking with $10 \%$ normal horse serum (NHS) in $0.3 \%$ Triton X-100 PBS (PBST) was performed before overnight incubation with biotinylated anti mouse $\operatorname{IgG}(1: 250$ in in $0.3 \%$ PBST, Vector Laboratories) at $4{ }^{\circ} \mathrm{C}$. Sections were then incubated with avidin-biotin-peroxidase complex, and colour-developed using a freshly prepared diaminobenzidine (DAB) solution. To ensure comparable DAB staining between sections is achieved, the time of the colour change was recorded, and DAB applied for each subsequent sample for same amount of time. 


\section{Immunohistochemistry}

Free-floating serial brain sections ( $30 \mu \mathrm{m}$ thick) were washed 3 times in PBS for 10min and blocked for $1 \mathrm{~h}$ in $10 \%$ normal goat serum (NGS) in $0.3 \%$ PBST. This was followed by an overnight incubation with primary antibody in $2 \%$ NGS in PBST at $4{ }^{\circ} \mathrm{C}$. The primary antibodies used in the study are explained in details below; Chicken antiGFAP (abcam AB4674, 1:500), Rabbit antilb1a (Wako 019-19741, 1:500 DF), Rat Anti-mouse CD31 (BD Pharmingen, 550274, 1:100), Mouse anti-mouse Cav1 (BD Biosciences, 1, 610407, 1:50 ), Mouse anti-mouse NeuN (Millipore, MAB377, 1:100), and Rabbit Anti-MBP (Abcam, ab40390, 1:200). After incubation with the primary antibodies sections were washed 3 times in PBS and incubated with fluorescently labelled secondary antibodies in $2 \%$ NGS in PBST. To visualise the primary antibodies, the following secondary antibodies were used; goat anti-Ck Alexa Fluor® 488 conjugate (Invitrogen A11039, 1:500), goat anti-Rabbit Alexa Fluor® 647 conjugate (Invitrogen, A21244 1:200), goat anti-mouse Alexa Fluor® 488 conjugate (Invitrogen A11001, 1:500), goat anti-Rat Alexa Fluor ${ }^{\circledR} 647$ conjugate (Invitrogen, A-21247, 1:200). At the end samples were washed 3 times in PBS and transferred on non-gelatine coated slides and left to dry overnight before slides were then coverslip with ProLong Gold Antifade Mountant with DAPI (Thermo Fischer Scientific, Inc., USA). Images were collected on either SP5 inverted microscope (for Cav1-1/CD31 \& NeuN/MBP) or SP8 inverted microscope (for Iba1/GFAP) using 63x objective in the striatum and the outer cortex at bregma $-0.58 \mathrm{~mm}$. Total microglia were counted and the percentage of DiI-Lp positive microglia recorded. The activation state of microglia was scored on activation scale of 0-3 based on their morphologies using a scoring system described before 75 .

\section{Data and statistical analyses}

Statistical analysis of the data was performed using Graph Pad Prism 7 software. Two-tailed unpaired student t-test and one-way analysis of variance followed by the Tukey multiple comparison test were used and p values $<0.05$ were considered significant. For all analyses, data are represented as mean \pm standard error of the mean (SEM), unless otherwise indicated.

\section{Associated Content}

\section{Supporting Information}

Supporting Information is available free of charge on the ACS Publications website

These include: Figure S1: Physicochemical characterisations of DiI-Lp, Figure S2:

Characterisation of ${ }^{111}$ In-Lp, Figure S3: IVIS Lumina II imaging of the brain with and without transcardiac perfusion, Figure S4: Selective liposomal accumulation into ischaemic cerebral cortex, Figure S5: Selective liposomal accumulation into the ipsilateral striatum $24 \mathrm{~h}$ after I.V administration, Figure S6: Confocal images of Cav-1 expression and DiI-Lp accumulation in contralateral striatum and contralateral cortex ( $2 \mathrm{~h}$ after DiI-Lp I.V administration), Figure S7: Confocal images of Cav-1 expression and DiI-Lp accumulation in contralateral striatum and contralateral cortex (24h after DiI-Lp I.V administration), Figure S8: Evaluation of DiI-Lp colocalisation with microglia and astrocytes in the ipsilateral cortex, Figure S9: Confocal images of DiI-Lp co-localisation with neurons and oligodendrocytes in the ipsilateral striatum, Figure S10: 
Confocal images of DiI-Lp co-localisation with neurons and oligodendrocytes in the ipsilateral cortex.

\section{AUTHOR INFORMATION \\ Corresponding Authors:}

Dr Zahraa Al-Ahmady email: zahraa.al-ahmady@ntu.ac.uk

Prof Stuart Allan email: stuart.allan@manchester.ac.uk

Prof Kostas Kostarelos email: kostas.kostarelos@manchester.ac.uk

\section{Author Contributions :}

ZA initiated, designed, planned, and led the study, performed almost all of the experimental work and data analysis, and drafted the manuscript. DJ performed SPECT/CT imaging and radiolabelling stability assays. SA Helped in the initial stages of the project with data analysis and IHC experiments. RY provided training on MCAo model and gave advice on the stroke model and data analysis throughout the project. MH performed CSF samples collections, in vitro uptake study and provided continuous advice on data analysis and interpretation. GC provided technical training on histological staining and cardiac perfusion. IS performed cranial window implantation and multiphoton imaging and data analysis. SA provided continuous guidance in the conceptual design of the work and data interpretation, reviewed and edited the manuscript. KK conceptualized the study, designed, planned, discussed the findings, reviewed, edited the manuscript, and overall supervised the work.

\section{Acknowledgments:}

The authors acknowledge Lipoid Co. (Germany) for the lipid sample gifts, the staff at the bioimaging facility at the University of Manchester for advice on confocal imaging and data analysis, and Aleksandr Mironov of the Faculty of Life Sciences EM Facility for his assistance with TEM experiment. They also wish to thank the Biological Services Facility at the University of Manchester for expert animal husbandry.

\section{References}

1. Westendorp, W. F.; Nederkoorn, P. J.; Vermeij, J. D., Post-Stroke Infection: a Systematic Review and Meta-Analysis. BMC Neurol. 2011, 11, 1-7.

2. Association, S. State of the nation. https://www.stroke.org.uk/sites/default/files/state_of the nation_2017 final_1.pdf (accessed 2017).

3. Tawil, S. E.; Muir, K. W., Thrombolysis and Thrombectomy for Acute Ischaemic Stroke. Clin. Med. 2017, 17 (2), 161-165.

4. Wahlgren, N. G.; Ahmed, N., Neuroprotection in Cerebral Ischaemia: Facts and Fancies - The Need for New Approaches. Cerebrovasc. Dis. 2004, 17(suppl 1) (Suppl. 1), 153-166.

5. Sutherland, B. A.; Minnerup, J.; Balami, J. S.; Arba, F.; Buchan, A. M.; Kleinschnitz, C., Neuroprotection for Ischaemic Stroke: Translation from the Bench to the Bedside. Int J Stroke. 2012, 7 (5), 407-418.

6. Sydserff, S. G.; Borelli, A. R.; Green, A. R.; Cross, A. J., Effect of NXY-059 on Infarct Volume After Transient or Permanent Middle Cerebral Artery Occlusion in the Rat; Studies on Dose, Plasma Concentration and Therapeutic Time Window. Br. J. Pharmacol. 2002, 135 (1), 103-112.

7. Al-Ahmady, Z. S., Selective Drug Delivery Approaches to Lesioned Brain Through Blood Brain Barrier Disruption. Expert Opin. Drug Delivery 2018, 1, 1-15. 
8. McColl, B. W.; Rothwell, N. J.; Allan, S. M., Systemic Inflammation Alters the Kinetics of Cerebrovascular Tight Junction Disruption After Experimental Stroke in Mice. J. Neurosci. 2008, 28 (38), 94519462 .

9. $\quad$ Knowland, D.; Arac, A.; Sekiguchi, K. J.; Hsu, M.; Lutz, S. E.; Perrino, J.; Steinberg, G. K.; Barres, B. A.; Nimmerjahn, A.; Agalliu, D., Stepwise Recruitment of Transcellular and Paracellular Pathways Underlies BloodBrain Barrier Breakdown in Stroke. Neuron 2014, 82 (3), 603-617.

10. Haley, M. J.; Lawrence, C. B., The Blood-Brain Barrier After Stroke: Structural Studies and the Role of Transcytotic Vesicles. J. Cereb. Blood Flow Metab. 2016, 28, 456-470.

11. Lorberboym, M.; Lampl, Y.; Sadeh, M., Correlation of 99mTc-DTPA SPECT of the Blood-Brain Barrier with Neurologic Outcome After Acute Stroke. J. Nucl. Med. 2003, 44 (12), 1898-1904.

12. Brouns, R.; Wauters, A.; De Surgeloose, D.; Mariën, P.; De Deyn, P. P., Biochemical Markers for BloodBrain Barrier Dysfunction in Acute Ischemic Stroke Correlate with Evolution and Outcome. Eur. Neurol. 2011, 65 (1), 23-31.

13. Rosenberg, G. A.; Yang, Y., Vasogenic Edema Due to Tight Junction Disruption by Matrix Metalloproteinases in Cerebral Ischemia. Neurosurg Focus. 2007, 22 (5), 1-5.

14. Lakhan, S. E.; Kirchgessner, A.; Tepper, D.; Leonard, A., Matrix Metalloproteinases and Blood-Brain Barrier Disruption in Acute Ischemic Stroke. Front Neurol 2013, 4 (32), 1-15.

15. Arai, K.; Lok, J.; Guo, S.; Hayakawa, K.; Xing, C.; Lo, E. H., Cellular Mechanisms of Neurovascular Damage and Repair after Stroke. J. Child Neurol. 2011, 26 (9), 1193-1198.

16. Prakash, R.; Carmichael, S. T., Blood-Brain Barrier Breakdown and Neovascularization Processes after Stroke and Traumatic Brain Injury. Curr. Opin. Neurol. 2015, 28 (6), 556-564.

17. Kang, E. J.; Major, S.; Jorks, D.; Reiffurth, C.; Offenhauser, N.; Friedman, A.; Dreier, J. P., Blood-Brain Barrier Opening to Large Molecules Does not Imply Blood-Brain Barrier Opening to Small Ions. Neurobiol. Dis. 2013, 52, 204-218.

18. Gursoy-Ozdemir, Y.; Yemisci, M.; Dalkara, T., Microvascular protection is essential for successful neuroprotection in stroke. J Neurochem 2012, 2, 2-11.

19. Maeda, H., The Enhanced Permeability and Retention (EPR) Effect in Tumor Vasculature: The Key Role of Tumor-Selective Macromolecular Drug Targeting. Adv. Enzyme Regul. 2001, 41, 189-207.

20. Allen, T. M.; Cullis, P. R., Liposomal Drug Delivery Systems: from Concept to Clinical Applications. Adv. Drug Delivery Rev. 2013, 65 (1), 36-48.

21. Hobbs, S. K.; Monsky, W. L.; Yuan, F.; Roberts, W. G.; Griffith, L.; Torchilin, V. P.; Jain, R. K., Regulation of Transport Pathways in Tumor Vessels: role of Tumor Type and Microenvironment. Proc. Natl. Acad. Sci. U. S. A. 1998, 95 (8), 4607-4612.

22. Huang, S. K.; Martin, F. J.; Jay, G.; Vogel, J.; Papahadjopoulos, D.; Friend, D. S., Extravasation and Transcytosis of Liposomes in Kaposi's Sarcoma-Like Dermal Lesions of Transgenic Mice Bearing the HIV tat Gene. Am. J. Pathol. 1993, 143 (1), 10-14.

23. Toyota, H.; Asai, T.; Oku, N., Process Optimization by Use of Design of Experiments: Application for Liposomalization of FK506. Eur. J. Pharm. Sci. 2017, 102, 196-202.

24. Kawaguchi, A. T.; Yamano, M.; Haida, M.; Ohba, H.; Kakiuchi, T.; Tsukada, H., Effect of Oxygen Affinity of Liposome-Encapsulated Hemoglobin on Cerebral Ischemia and Reperfusion as Detected by Positron Emission Tomography in Nonhuman Primates. Artif. Organs 2017, 41 (4), 336-345.

25. Kawaguchi, A. T.; Haida, M.; Ohba, H.; Yamano, M.; Fukumoto, D.; Tsukada, H., Liposome-Encapsulated Hemoglobin Ameliorates Ischemic Stroke in Nonhuman Primates: longitudinal Observation. Artif Organs 2013, 37 (10), 904-912.

26. Ishii, T.; Asai, T.; Oyama, D.; Fukuta, T.; Yasuda, N.; Shimizu, K.; Minamino, T.; Oku, N., Amelioration of Cerebral Ischemia-Reperfusion Injury Based on Liposomal Drug Delivery System with Asialo-Erythropoietin. $J$. Controlled Release 2012, 160 (1), 81-87.

27. Fukumoto, D.; Kawaguchi, A. T.; Haida, M.; Yamano, M.; Ogata, Y.; Tsukada, H., LiposomeEncapsulated Hemoglobin Reduces the Size of Cerebral Infarction in Rats: Effect of Oxygen Affinity. Artif. Organs 2009, 33 (2), 159-163.

28. Adibhatla, R. M.; Hatcher, J. F.; Tureyen, K., CDP-Choline Liposomes Provide Significant Reduction in Infarction Over Free CDP-Choline in Stroke. Brain Res. 2005, 5, 1-2.

29. Barenholz, Y., Doxil ${ }^{\circledR}$ - The First FDA-Approved Nano-Drug: Lessons Learned. J. Controlled Release 2012, 160 (2), 117-134. 
30. Jiao, X.; He, P.; Li, Y.; Fan, Z.; Si, M.; Xie, Q.; Chang, X.; Huang, D., The Role of Circulating Tight Junction Proteins in Evaluating Blood Brain Barrier Disruption following Intracranial Hemorrhage. Dis. Markers 2015, 860120 (10), 1-13.

31. Jiao, H.; Wang, Z.; Liu, Y.; Wang, P.; Xue, Y., Specific Role of Tight Junction Proteins Claudin-5, Occludin, and ZO-1 of the Blood-Brain Barrier in a Focal Cerebral Ischemic Insult. J. Mol. Neurosci. 2011, 44 (2), 130-139.

32. Gumbleton, M.; Abulrob, A. G.; Campbell, L., Caveolae: an alternative membrane transport compartment. Pharm Res 2000, 17 (9), 1035-1048.

33. Nag, S.; Venugopalan, R.; Stewart, D. J., Increased Caveolin-1 Expression Precedes Decreased Expression of Occludin and Claudin-5 During Blood-Brain Barrier Breakdown. Acta Neuropathol. 2007, 114 (5), 459-469.

34. Krueger, M.; Hartig, W.; Reichenbach, A.; Bechmann, I.; Michalski, D., Blood-brain barrier breakdown after embolic stroke in rats occurs without ultrastructural evidence for disrupting tight junctions. PloS One 2013, 8 (2), 1-11.

35. Cipolla, M. J.; Crete, R.; Vitullo, L.; Rix, R. D., Transcellular transport as a mechanism of blood-brain barrier disruption during stroke. Front Biosci 2004, 9, 777-785.

36. Nahirney, P. C.; Reeson, P.; Brown, C. E., Ultrastructural analysis of blood-brain barrier breakdown in the peri-infarct zone in young adult and aged mice. J Cereb Blood Flow Metab 2016, 36 (2), 413-425.

37. Willis, C. L., Glia-induced reversible disruption of blood-brain barrier integrity and neuropathological response of the neurovascular unit. Toxicol Pathol 2011, 39 (1), 172-185.

38. A. Burgess, K. H., Drug delivery across the blood-brain barrier using focused ultrasound. Expert Opin. Drug Delivery 2014, 11, 711-721.

39. Voigt, J.; Christensen, J.; Shastri, V. P., Differential Uptake of Nanoparticles by Endothelial Cells Through Polyelectrolytes with Affinity for Caveolae. Proc. Natl. Acad. Sci. U. S. A. 2014, 111 (8), 2942-2947.

40. $\quad$ Takeuchi, K.; Morizane, Y.; Kamami-Levy, C.; Suzuki, J.; Kayama, M.; Cai, W.; Miller, J. W.; Vavvas, D. G., AMP-dependent kinase inhibits oxidative stress-induced caveolin-1 phosphorylation and endocytosis by suppressing the dissociation between c-Abl and Prdx1 proteins in endothelial cells. J Biol Chem 2013, 288 (28), 20581-20591.

41. Andreone, B. J.; Chow, B. W.; Tata, A.; Lacoste, B.; Ben-Zvi, A.; Bullock, K.; Deik, A. A.; Ginty, D. D.; Clish, C. B.; Gu, C., Blood-Brain Barrier Permeability Is Regulated by Lipid Transport-Dependent Suppression of Caveolae-Mediated Transcytosis. Neuron 2017, 94 (3), 581-594.

42. Nimmerjahn, A.; Kirchhoff, F.; Helmchen, F., Resting Microglial Cells are Highly Dynamic Surveillants of Brain Parenchyma in vivo. Science 2005, 308 (5726), 1314-1318.

43. $\quad$ Streit, W. J.; Walter, S. A.; Pennell, N. A., Reactive microgliosis. Prog Neurobiol 1999, 57 (6), 563-581.

44. Ishii, T.; Asai, T.; Oyama, D.; Agato, Y.; Yasuda, N.; Fukuta, T.; Shimizu, K.; Minamino, T.; Oku, N., Treatment of cerebral ischemia-reperfusion injury with PEGylated liposomes encapsulating FK506. Faseb J 2013, 27 (4), 1362-1370.

45. $\quad$ Fukuta, T.; Asai, T.; Sato, A.; Namba, M.; Yanagida, Y.; Kikuchi, T.; Koide, H.; Shimizu, K.; Oku, N., Neuroprotection Against Cerebral Ischemia/Reperfusion Injury by Intravenous Administration of Liposomal Fasudil. Int. J. Pharm. 2016, 506 (1-2), 129-137.

46. Sandoval, K. E.; Witt, K. A., Blood-brain barrier tight junction permeability and ischemic stroke. Neurobiol Dis 2008, 32 (2), 200-219.

47. Dore-Duffy, P.; Owen, C.; Balabanov, R.; Murphy, S.; Beaumont, T.; Rafols, J. A., Pericyte migration from the vascular wall in response to traumatic brain injury. Microvasc Res 2000, 60 (1), 55-69.

48. Liu, S.; Agalliu, D.; Yu, C.; Fisher, M., The role of pericytes in blood-brain barrier function and stroke. Curr Pharm Des 2012, 18 (25), 3653-3662.

49. Armulik, A.; Genove, G.; Mae, M.; Nisancioglu, M. H.; Wallgard, E.; Niaudet, C.; He, L.; Norlin, J.; Lindblom, P.; Strittmatter, K.; Johansson, B. R.; Betsholtz, C., Pericytes regulate the blood-brain barrier. Nature 2010, 468 (7323), 557-61.

50. Daneman, R.; Zhou, L.; Kebede, A. A.; Barres, B. A., Pericytes are required for blood-brain barrier integrity during embryogenesis. Nature 2010, 468 (7323), 562-566.

51. Simionescu, M.; Popov, D.; Sima, A., Endothelial transcytosis in health and disease. Cell Tissue Res 2009, 335 (1), 27-40.

52. Lossinsky, A. S.; Shivers, R. R., Structural pathways for macromolecular and cellular transport across the blood-brain barrier during inflammatory conditions. Review. Histol Histopathol 2004, 19 (2), 535-564.

53. Arai, K.; Jin, G.; Navaratna, D.; Lo, E. H., Brain angiogenesis in developmental and pathological processes: neurovascular injury and angiogenic recovery after stroke. Febs $J \mathbf{2 0 0 9 ,} 276$ (17), 4644-4652. 
54. del Zoppo, G. J., Inflammation and the neurovascular unit in the setting of focal cerebral ischemia. Neuroscience 2009, 158 (3), 972-982.

55. Shi, Y.; Zhang, L.; Pu, H.; Mao, L.; Hu, X.; Jiang, X.; Xu, N.; Stetler, R. A.; Zhang, F.; Liu, X.; Leak, R. K.; Keep, R. F.; Ji, X.; Chen, J., Rapid endothelial cytoskeletal reorganization enables early blood-brain barrier disruption and long-term ischaemic reperfusion brain injury. Nat Commun 2016, 7 (10523), 1-18.

56. Park, K. P.; Rosell, A.; Foerch, C.; Xing, C.; Kim, W. J.; Lee, S.; Opdenakker, G.; Furie, K. L.; Lo, E. H., Plasma and brain matrix metalloproteinase-9 after acute focal cerebral ischemia in rats. Stroke 2009, 40 (8), 28362842 .

57. Allan, S. M.; Tyrrell, P. J.; Rothwell, N. J., Interleukin-1 and neuronal injury. Nat Rev Immunol 2005, 5 (8), 629-640.

58. Giles, J. A.; Greenhalgh, A. D.; Davies, C. L.; Denes, A.; Shaw, T.; Coutts, G.; Rothwell, N. J.; McColl, B. W.; Allan, S. M., Requirement for interleukin-1 to drive brain inflammation reveals tissue-specific mechanisms of innate immunity. Eur J Immunol 2015, 45 (2), 525-530.

59. Fumagalli, S.; Perego, C.; Pischiutta, F.; Zanier, E. R.; De Simoni, M. G., The Ischemic Environment Drives Microglia and Macrophage Function. Front. Neurol. 2015, 6 (81), 1-19.

60. Pradillo, J. M.; Denes, A.; Greenhalgh, A. D.; Boutin, H.; Drake, C.; McColl, B. W.; Barton, E.; Proctor, S. D.; Russell, J. C.; Rothwell, N. J.; Allan, S. M., Delayed administration of interleukin-1 receptor antagonist reduces ischemic brain damage and inflammation in comorbid rats. J Cereb Blood Flow Metab 2012, 32 (9), 1810-1019. 61. Maysami, S.; Wong, R.; Pradillo, J. M.; Denes, A.; Dhungana, H.; Malm, T.; Koistinaho, J.; Orset, C.; Rahman, M.; Rubio, M.; Schwaninger, M.; Vivien, D.; Bath, P. M.; Rothwell, N. J.; Allan, S. M., A cross-laboratory preclinical study on the effectiveness of interleukin-1 receptor antagonist in stroke. J Cereb Blood Flow Metab 2016, 36 (3), 596-605.

62. $\quad$ Singh, N.; Hopkins, S. J.; Hulme, S.; Galea, J. P.; Hoadley, M.; Vail, A.; Hutchinson, P. J.; Grainger, S.; Rothwell, N. J.; King, A. T.; Tyrrell, P. J., The effect of intravenous interleukin-1 receptor antagonist on inflammatory mediators in cerebrospinal fluid after subarachnoid haemorrhage: a phase II randomised controlled trial. J Neuroinflammation 2014, 11 (1), 1742-2094.

63. $\quad$ Hu, X.; Leak, R. K.; Shi, Y.; Suenaga, J.; Gao, Y.; Zheng, P.; Chen, J., Microglial and macrophage polarization-new prospects for brain repair. Nat Rev Neurol. 2015, 11 (1), 56-64.

64. Wong, R.; Gibson, C. L.; Kendall, D. A.; Bath, P. M., Evaluating the Translational Potential of Progesterone Treatment following Transient Cerebral Ischaemia in Male Mice. BMC Neurosci. 2014, 15 (131), 0140131 .

65. Clark, W.; Gunion-Rinker, L.; Lessov, N.; Hazel, K., Citicoline Treatment for Experimental Intracerebral Hemorrhage in Mice. Stroke 1998, 29 (10), 2136-2140.

66. Al-Ahmady, Z. S.; Chaloin, O.; Kostarelos, K., Monoclonal Antibody-Targeted, Temperature-Sensitive Liposomes: In vivo Tumor Chemotherapeutics in Combination with Mild Hyperthermia. J. Controlled Release 2014, 196, 332-343.

67. Al-Ahmady; Scudamore; Kostarelos, Triggered Doxorubicin Release in Solid Tumors from Thermosensitive Liposome-Peptide Hybrids: Critical Parameters and Therapeutic Efficacy. Int. J. Cancer 2015, 137 (3), 731-743.

68. Al-Ahmady, Z. S.; Al-Jamal, W. T.; Bossche, J. V.; Bui, T. T.; Drake, A. F.; Mason, A. J.; Kostarelos, K., Lipid-Peptide Vesicle Nanoscale Hybrids for Triggered Drug Release by Mild Hyperthermia in vitro and in vivo. ACS Nano 2012, 6 (10), 9335-9346.

69. Goldey, G. J.; Roumis, D. K.; Glickfeld, L. L.; Kerlin, A. M.; Reid, R. C.; Bonin, V.; Schafer, D. P.; Andermann, M. L., Removable cranial windows for long-term imaging in awake mice. Nat Protoc 2014, 9 (11), 2515-2538.

70. Echarri, A.; Del Pozo, M. A., Caveolae - mechanosensitive membrane invaginations linked to actin filaments. J Cell Sci 2015, 128 (15), 2747-58.

71. Henley, J. R.; Krueger, E. W.; Oswald, B. J.; McNiven, M. A., Dynamin-mediated internalization of caveolae. J Cell Biol 1998, 141 (1), 85-99.

72. Guo, P.; Liu, D.; Subramanyam, K.; Wang, B.; Yang, J.; Huang, J.; Auguste, D. T.; Moses, M. A., Nanoparticle elasticity directs tumor uptake. Nat Commun 2018, 9 (1), 017-02588.

73. Maysami, S.; Haley, M. J.; Gorenkova, N.; Krishnan, S.; McColl, B. W.; Lawrence, C. B., Prolonged DietInduced Obesity in Mice Modifies the Inflammatory Response and Leads to Worse Outcome After Stroke. J. Neuroinflammation 2015, 12 (140), 1-12. 
74. Osborne, K. A.; Shigeno, T.; Balarsky, A. M.; Ford, I.; McCulloch, J.; Teasdale, G. M.; Graham, D. I., Quantitative Assessment of Early Brain Damage in a Rat model of Focal Cerebral Cschaemia. J. Neurol.,

Neurosurg. Psychiatry 1987, 50 (4), 402-410.

75. Daniels, M. J.; Rivers-Auty, J.; Schilling, T.; Spencer, N. G.; Watremez, W.; Fasolino, V.; Booth, S. J.; White, C. S.; Baldwin, A. G.; Freeman, S.; Wong, R.; Latta, C.; Yu, S.; Jackson, J.; Fischer, N.; Koziel, V.; Pillot, T.; Bagnall, J.; Allan, S. M.; Paszek, P., et al., Fenamate NSAIDs inhibit the NLRP3 Inflammasome and Protect Against Alzheimer's Disease in Rodent Models. Nat. Commun. 2016, 7 (12504), 1-10. 
Figures:

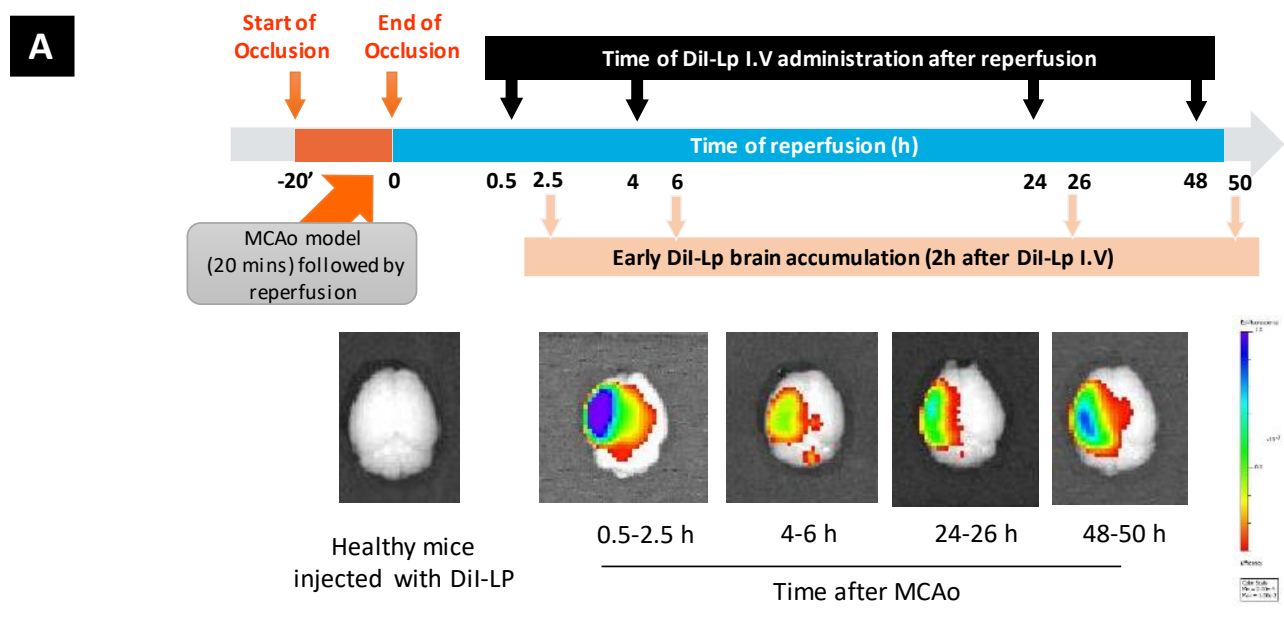

B

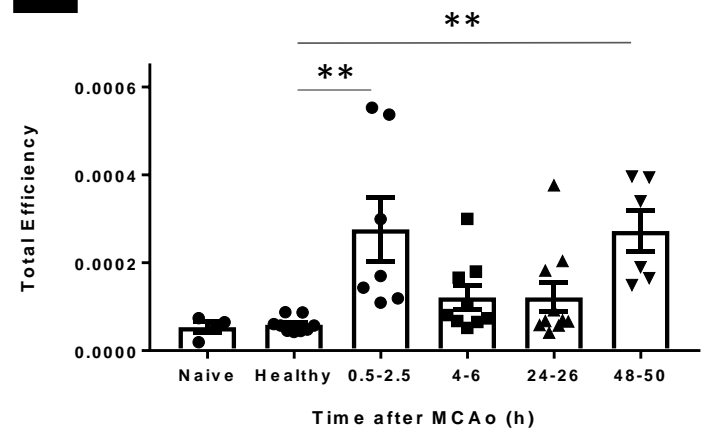

C

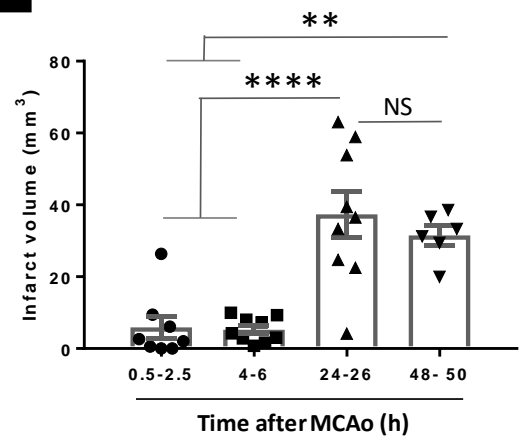

D

Healthy mice

injected with Dil-Lp
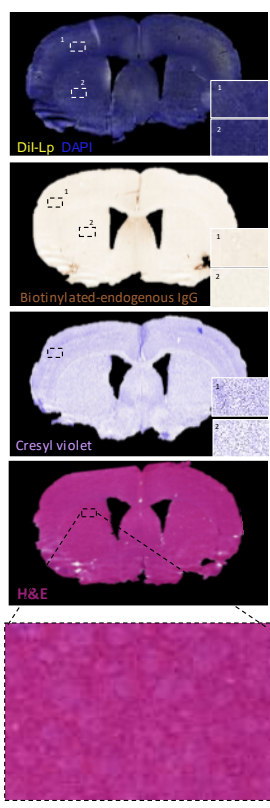
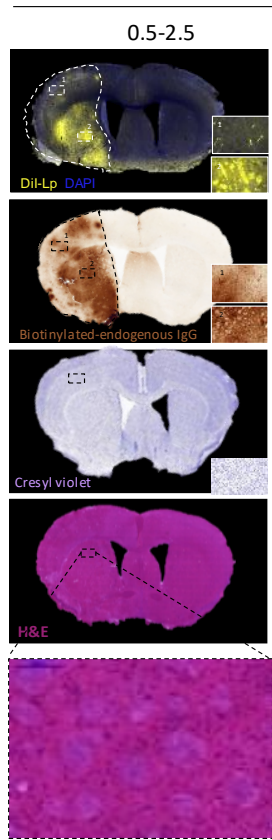

Time after MCAo

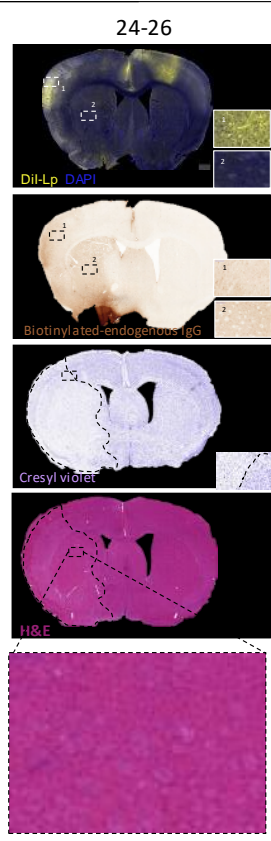

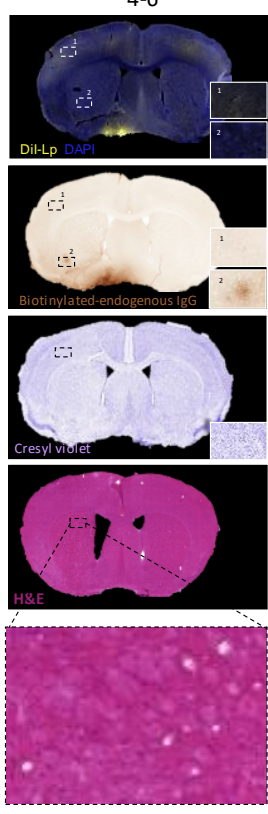
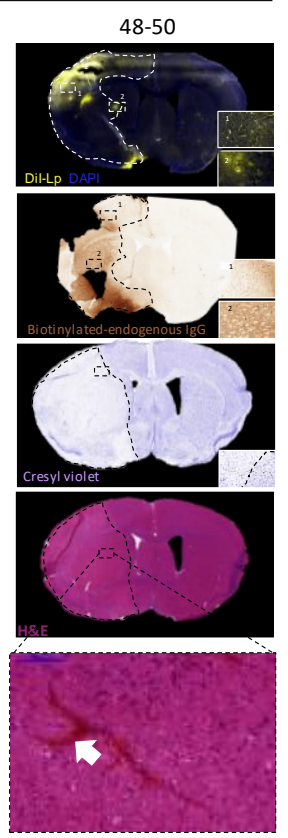
Figure 1: Selective liposomal accumulation into the ischaemic brain shortly after Lp-DiI I.V administration. Selective recruitments of DiI-Lp into the ischaemic region of the brain in MCAo model of stroke were studies by (A) IVIS Lumina II imaging system showing the selective accumulation of liposomes into the ischaemic left side of brain as early as $2 \mathrm{~h}$ after DiI-Lp I.V administration. Minimum DiI-Lp signal was detected from the contralateral side of the brain (right) and healthy mice (naive mice injected with liposomes). (B) Quantification of the fluorescent single of DiI-Lp in the brain by IVIS Lumina imaging software was performed by drawing a region of interest (ROI) that covers the whole brain and expressed as total efficiency. Colour scale of epifluorescent signal range from $\mathrm{min}=2.00^{-4}$ (red) to $\max =1.00^{-3}$ (blue). A bi-phasic recruitment of DiI-Lp into the ischaemic area was observed showing significant increase when injected $0.5 \mathrm{~h}$ or 48h following MCAo and reperfusion. (C) Quantification of the volume of ischaemic damage was performed on representative sections taken at eight defined coronal levels. (D) Brain sections from healthy mice and mice after MCAo showing; maximum selective recruitment of DiI-Lp into ischaemic left side of the brain (marked by dashed white lines at $0.5-2.5 \mathrm{~h}$ and $48-50 \mathrm{~h}$ time point), compared to less liposomal accumulation at 4-6h and 24-26h groups and no liposomes accumulation into the healthy brain. Accumulation of liposomes into the ischaemic brain have a similar distribution to the endogenous IgG leakage into the brain (outline by dashed black lines) that is used as indication of BBB disruption. Cresyl violet staining of brain sections represents the extent of brain damage induced at different time points following 20min MCAo. In most cases moderate to extensive damage of both cortical and subcortical regions was observed at 1-2d post MCAo. Cerebral accumulation of DiI-Lp given at the early phase after reperfusion $(0.5 \mathrm{~h} \& 4 \mathrm{~h})$ were observed before clear histological evidence of neuronal damage was observed as indicated from the cresyl violet stain. H\&E images confirmed that liposomal accumulation was associated with rare instances of vessel lumen collapse (white arrows). Inset images represent $40 \mathrm{x}$ magnifications for DiI-Lp and 20x magnification for IgG, cresyl violet and H\&E. Statistical analysis of $\mathrm{B} \& \mathrm{C}$ was performed using one-way analysis of variance followed by the Tukey multiple comparison test and $p$ values $<0.05$ were considered significant. $n=6-9$ in each group. 


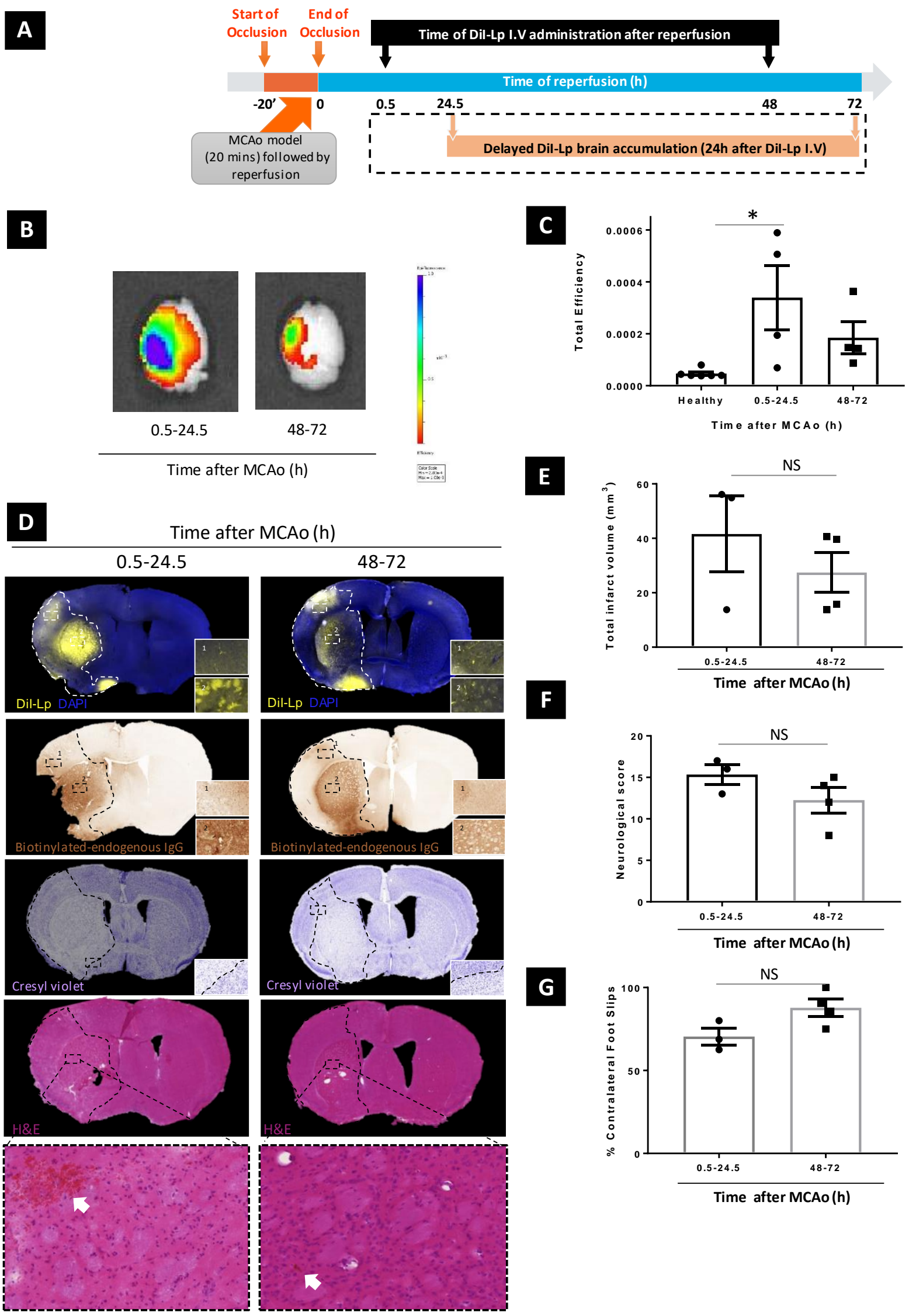


Figure 2: Selective liposomal accumulation into ischaemic brain $24 \mathrm{~h}$ after I.V administration. (A) Schematic presentation of the experimental time points used for DiI-Lp I.V administration following 20min MCAo and reperfusion. The time points of DiL-Lp intravenous injection illustrated the separate groups studied each received single injection. Selective recruitments of DiI-Lp into the ischaemic region of the brain in MCAo model of stroke were studies by (B) IVIS Lumina II imaging system showed that selective accumulation of liposomes into the ischaemic (left side) persists 24h after DiI-Lp I.V administration. (C) Quantification of the total fluorescent single of DiI-Lp in the brain $24 \mathrm{~h}$ after injection indicated that there was no change in liposomal accumulation when injected $0.5 \mathrm{~h}$ after MCAo, whereas reduced total liposomal level was observed when injected $48 \mathrm{~h}$ after MCAo. Colour scale of epi-fluorescent signal range from $\min =2.00^{-4}$ (red) to $\max =1.00^{-3}$ (blue). A bi-phasic recruitment of DiI-Lp into the ischaemic area was observed showing significant increase when injected $0.5 \mathrm{~h}$ or $48 \mathrm{~h}$ following MCAo and reperfusion. (D) Cerebral accumulations of DiI-liposomes administered $0.5 \mathrm{~h} \& 48 \mathrm{~h}$ after reperfusion were still observed in the ischaemic region $24 \mathrm{~h}$ afterwards and observed co-localised with BBB damage (endogenous IgG) and infarct area (Cresyl violet) with rare evidence of vessel lumen collapse $(\mathrm{H} \& \mathrm{E})$ indicated by white arrows. Inset images represent $40 \mathrm{x}$ magnifications for DiI-Lp and 20x magnification for IgG, cresyl violet and H\&E. Primary brain injury after MCAo was confirmed by (E) Quantification of volume of ischaemic damage on representative sections taken at eight defined coronal levels of brain tissues stained with cresyl violet, (F) measuring focal deficit scoring (0-28) and (G) foot-fault test. Statistical analysis of $\mathrm{C}$ was performed using oneway analysis of variance followed by the Tukey multiple comparison test, $n=4-6$ in each group. Two-tailed unpaired student t-test used for statistical analysis of the data in E,F \& G (n=3-4 in each group). $\mathrm{p}$ values $<0.05$ were considered significant. 


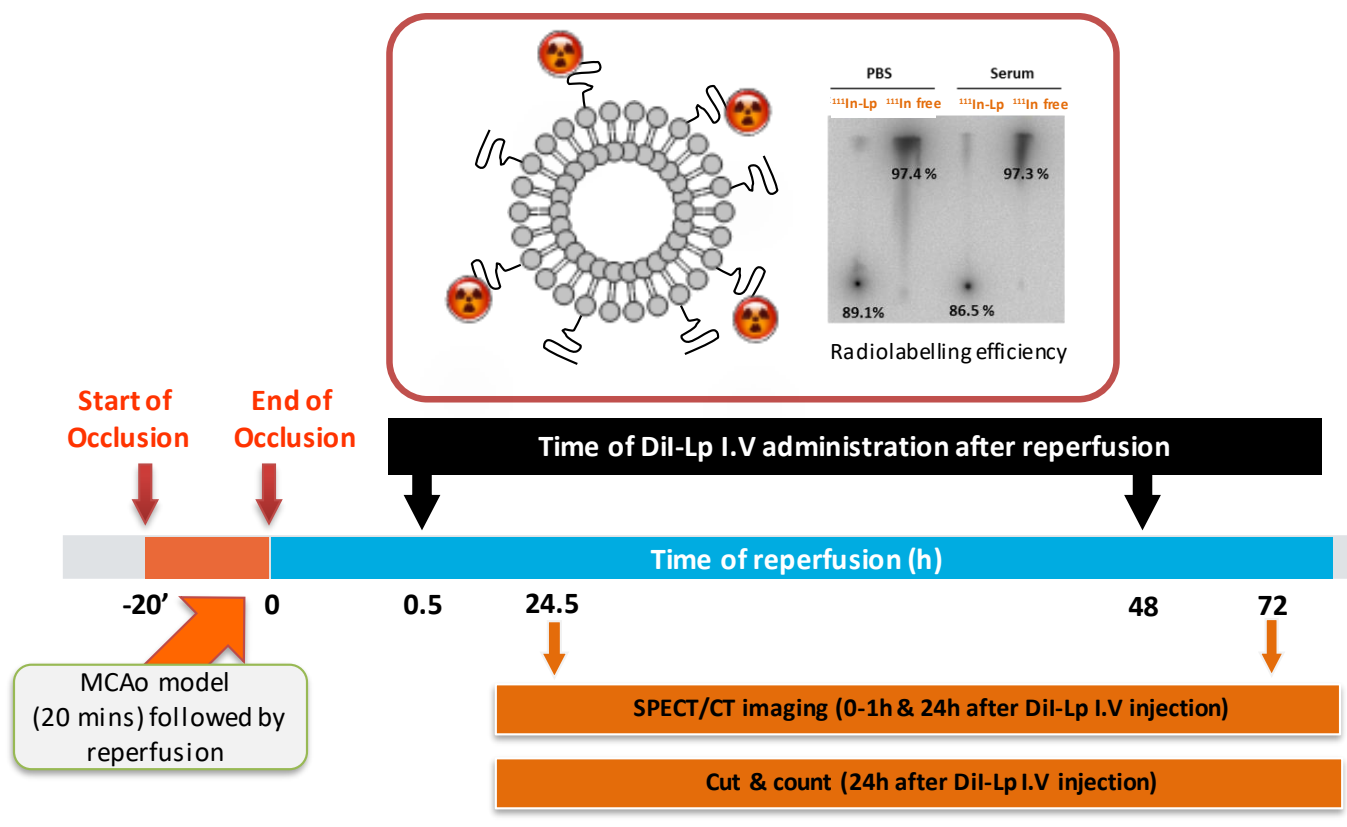

\section{B}

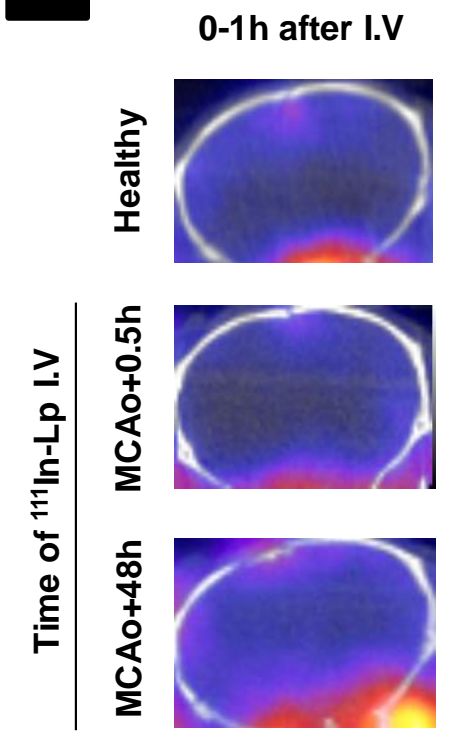

\section{4h after I.V}
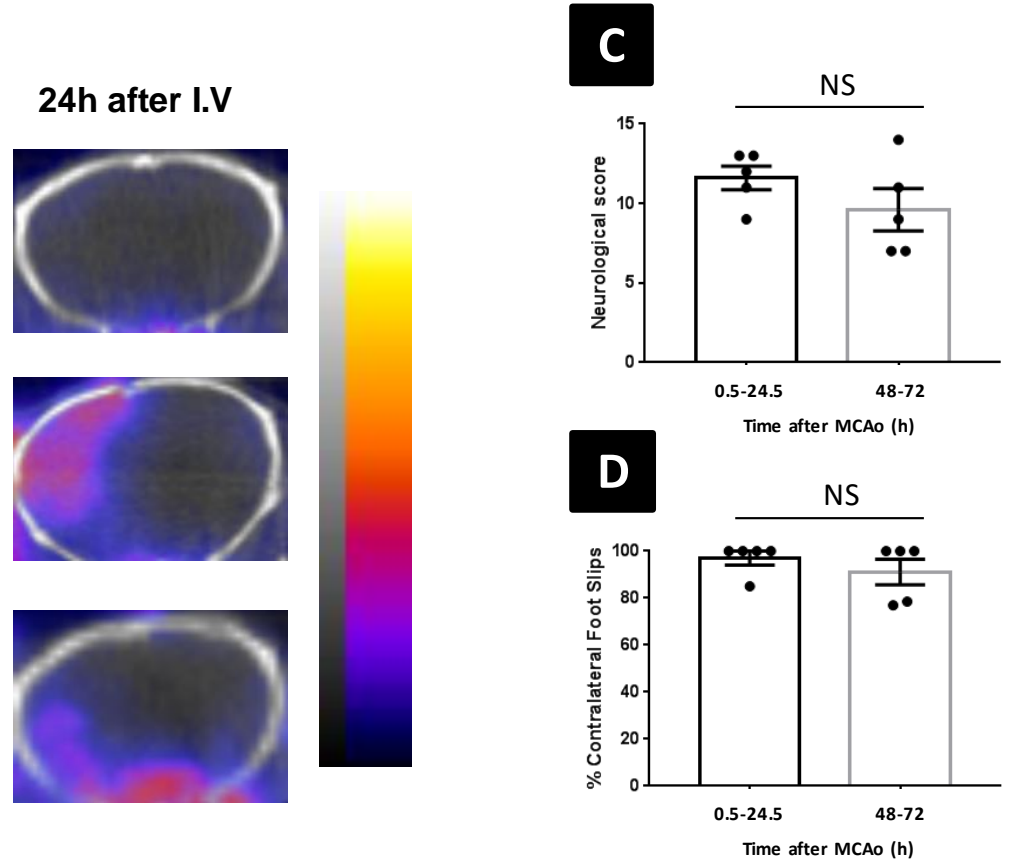

\section{E Ipsilateral}

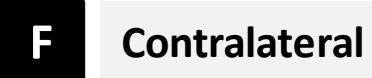

\section{G CSF}
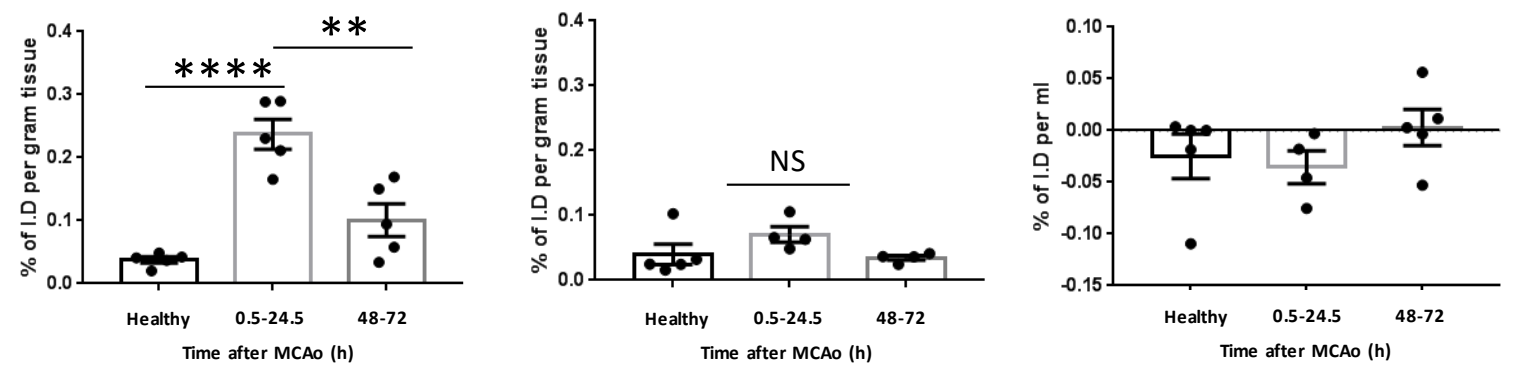
Figure 3: Quantification of liposomes accumulation into the brain after MCAo. (A) Schematic presentation of experimental design and ${ }^{111} \mathrm{In}$-Lp radiolabelling efficiency. To quantify and study the accumulation of liposomes into the brain in real-time SPECT/CT imaging and gamma counting of the liposomes were performed after radiolabelling with ${ }^{111} \mathrm{In}$. The immobile spot on the TLC strips indicated the percentage of radiolabelled ${ }^{111} \mathrm{In}-\mathrm{Lp}$, while free ${ }^{111} \mathrm{In}$ was detected as the mobile spots near the solvent front. Very minimum free ${ }^{111}$ In was detected in the ${ }^{111} \mathrm{In}-\mathrm{Lp}$ samples. The radiolabelling stabilities of the final product of ${ }^{111} \mathrm{In}-\mathrm{Lp}$ were studied at $37^{\circ} \mathrm{C}$ after incubation in PBS and 50\% serum for up to $48 \mathrm{~h}$. TLC Data indicated $>85 \%$ radiolabelling efficiency with excellent serum stability. The time points represent separate groups each received single injection of ${ }^{111} \mathrm{In}-\mathrm{Lp}$ intravenously. (B) Representative SPECT/CT imaging confirmed the selective accumulation of the liposomes into the ipsilateral side of the brain (left) compared to contralateral side. In the absence of ischaemic stroke, no accumulation of ${ }^{111} \mathrm{In}-\mathrm{Lp}$ was detected. Assessment of (C) neurological focal deficit scoring (0-28) and (D) foot-fault test showed no significant differences between $0.5 \mathrm{~h}(\mathrm{MCAo}+1 \mathrm{~d})$ and $48 \mathrm{~h}$ groups $(\mathrm{MCAo}+3 \mathrm{~d})$. Quantification of ${ }^{111} \mathrm{In}-\mathrm{Lp}$ accumulation into (E) ipsilateral brain side and (F) contralateral brain side. Values are expressed as $\%$ of I.D \pm SEM per gram brain tissue. Data confirmed significant increase in selective brain liposomal level when injected at $0.5 \mathrm{~h}$ after MCAo and to a less extent when given $48 \mathrm{~h}$ after MCAo. (G) Detection of ${ }^{111} \mathrm{In}-\mathrm{Lp}$ in the CSF after MCAo indicated no significant differences in CSF liposomal level compared to healthy control group. The data in $\mathrm{C} \& \mathrm{D}$ were analysed by two-tailed unpaired student t-test $(n=3-4)$, where as one-way analysis of variance followed by the Tukey multiple comparison test $(\mathrm{n}=4-5)$ was used for graph $\mathrm{E}, \mathrm{F} \& \mathrm{G}$. The data were considered significant if $\mathrm{p}$ values $<0.05$. 


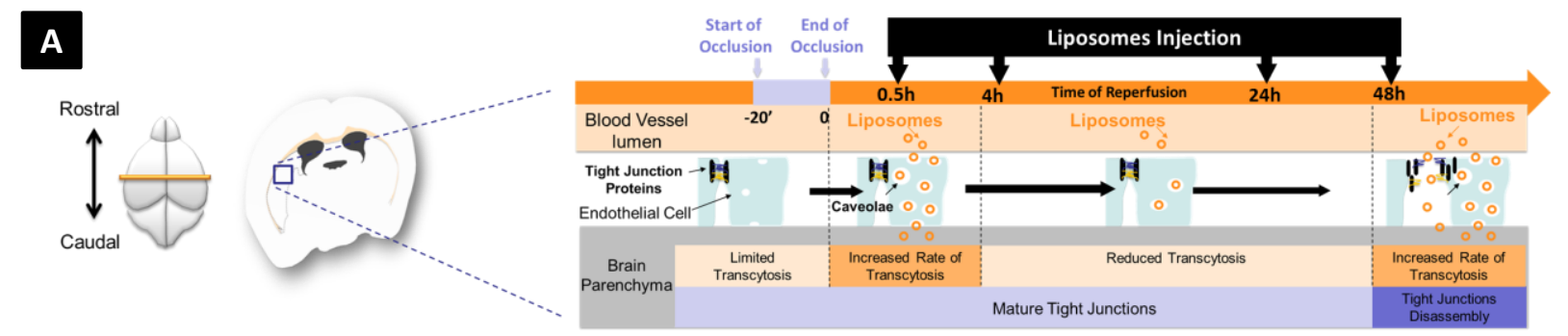

\section{B}
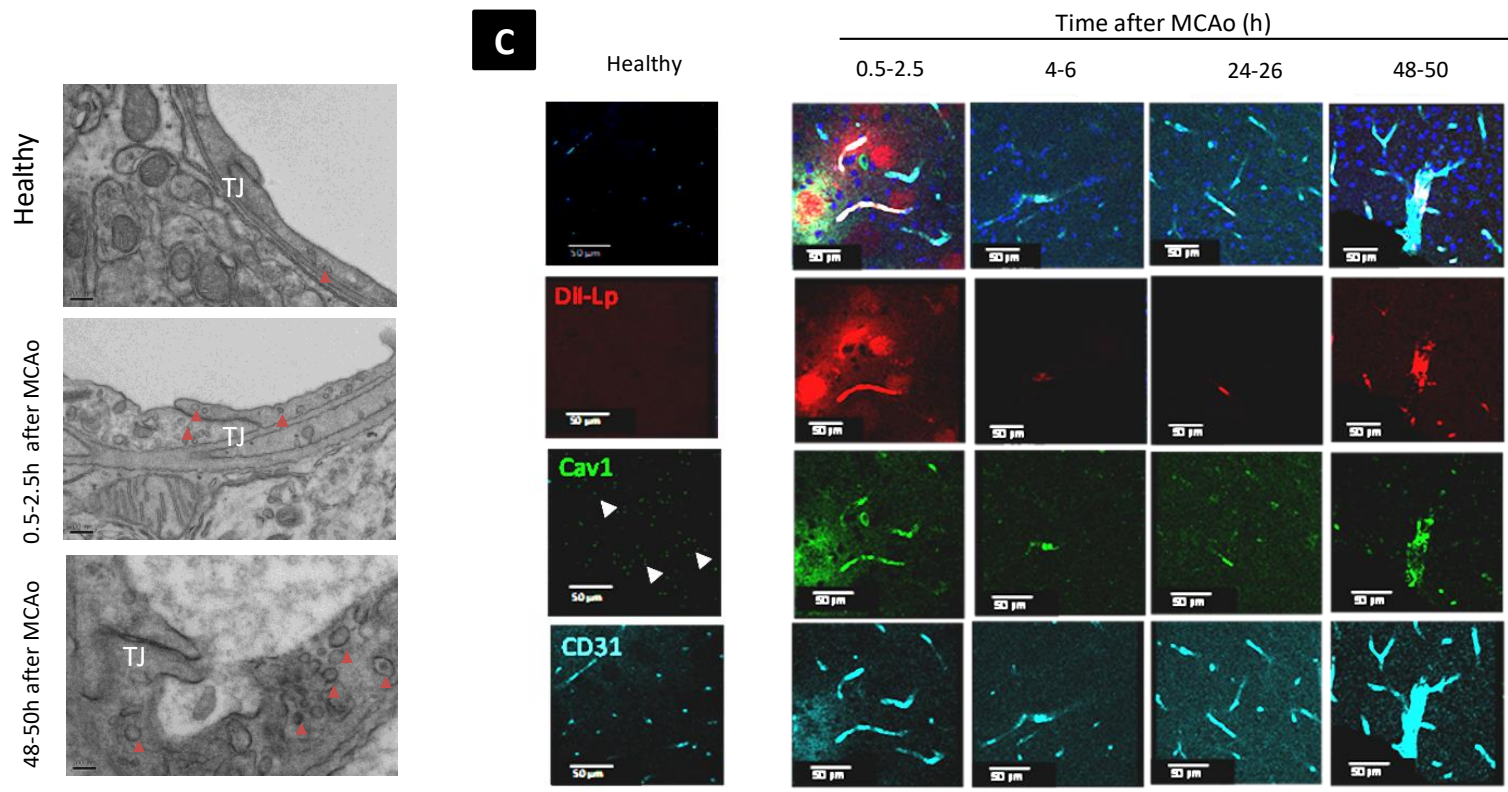

D

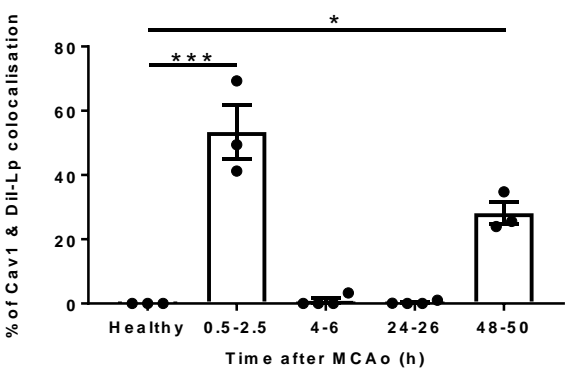

$\mathbf{E}$

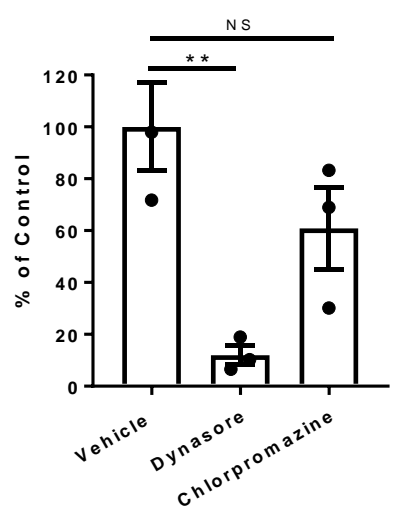

\section{$\mathbf{F}$}
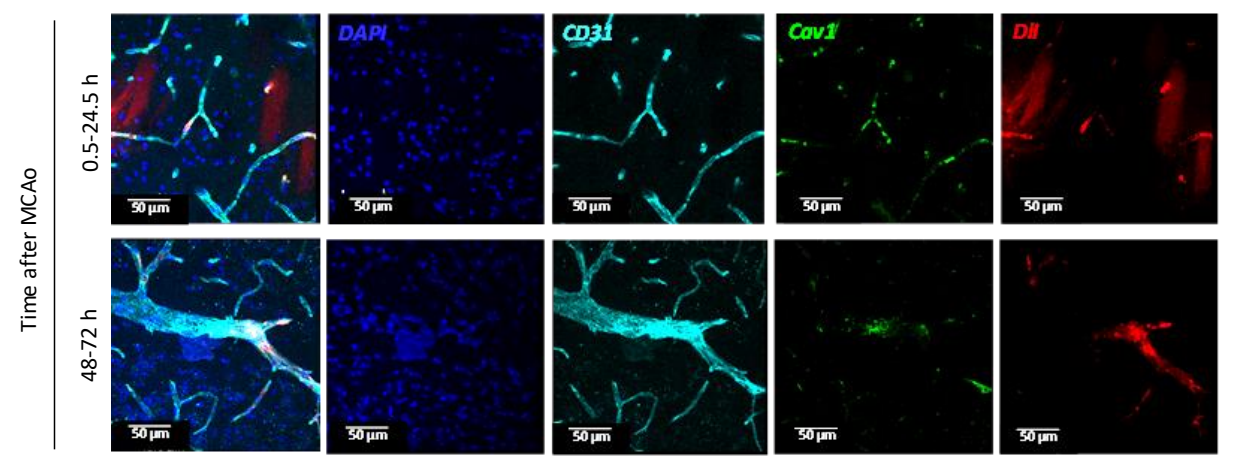

Figure 4: Mechanism of selective liposomal accumulation into ischaemic brain. (A) Schematic presentation of the experimental plan and the time frame for DiI-Lp intravenous administration 
after 20min of MCAo model in mice. The time points represent separate groups each received single injection of DiI-Lp intravenously. The two possible mechanisms of selective liposome recruitment into the ischaemic brain are elucidated. (B) Representative TEM images confirmed ultrastructural changes in endothelial cells after MCAo compared to healthy mice. Amplification of transcytotic vesicles (indicated by red arrow head) number was clearly evident at $0.5-2.5 \mathrm{~h}$ and 48-50h after MCAo. Enlargement of the vesicles and ultrastructural changes to endothelial cells TJs (protrusions and change in morphology) were observed at late but not early time points after MCAo. (C) Representative images demonstrating immunofluorescence labelling of endothelial CD31 (cyan) and Cav-1 (green) markers in the striatum at $-0.58 \mathrm{~mm}$ from the bregma. White triangles represent areas of low Cav1 expression in healthy brain. Analysis was performed $2 \mathrm{~h}$ after I.V injection of DiI-Lp for each group. A biphasic increase in Cav-1 immunofluorescence was observed for $0.5-2.5 \mathrm{~h}$ and $48-50 \mathrm{~h}$ groups that co-localises with the areas of DiI-Lp leakage into the ischaemic brain. On the contrary, Cav-1 expression and accumulation of DiI-Lp in the brain were minimum for 4-6h and 24-26h groups. (D) Quantitative analysis of Cav1 co-localisation with DiI-Lp $2 \mathrm{~h}$ after I.V injection confirmed the biphasic pattern observed from confocal images. (E) Uptake of DiI-Lp by hCMEC/D3 brain endothelial cells in vitro expressed as \% of control cells treated with vehicle showed a significant inhibition of DiI-Lp uptake in the presence of dynasore. (F) Evaluation of DiI-Lp leakage into the ischaemic brain 24h following I.V into MCAo mice at $0.5 \mathrm{~h}$ and $48 \mathrm{~h}$ following reperfusion. Co-localisation of DiI-Lp with areas of enhanced Cav-1 expression were detected. Cardiac perfusion was performed to remove any DiI-Lp from the circulation. Statistical analysis of the results in D was performed using one-way analysis of variance followed by the Tukey multiple comparison test, $n=3-4$ in each group. $p$ values $<0.05$ were considered significant. 

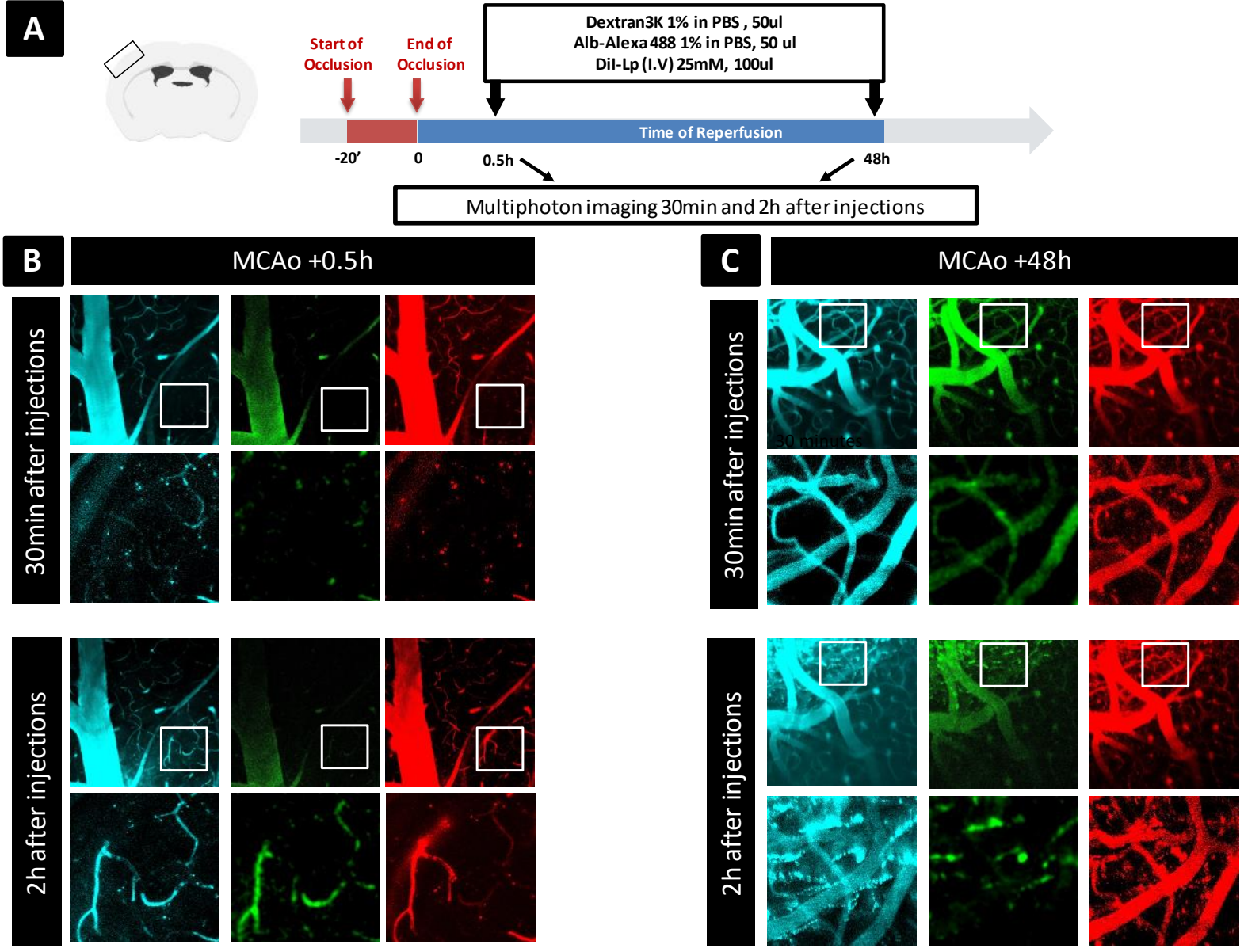

D Dextran Albumin Dil-Lp

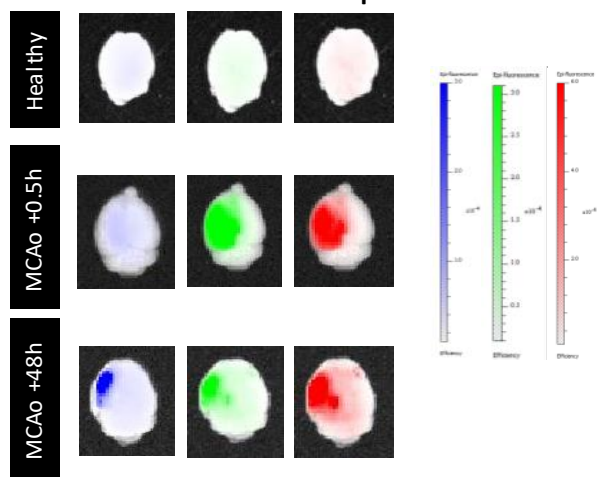

\section{E}
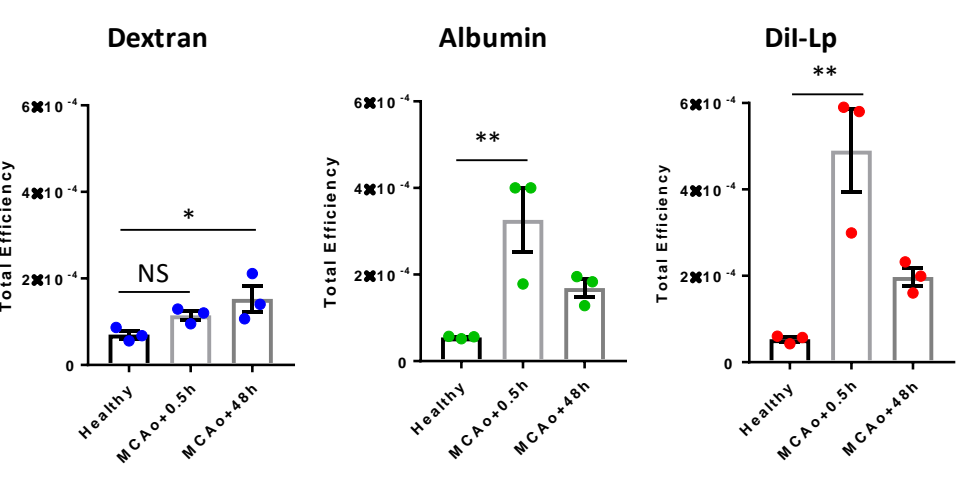

Figure 5: Co-localisation of liposomal accumulation into ischemic brain with transcellular and paracellular markers. (A) schematic presentation of study design for multiphoton imaging. In this study each mouse received I.V injections of DiI Lp $(25 \mathrm{mM}, 100 \mu \mathrm{L})$, cascade blue dextran $3 \mathrm{~K}(50 \mu \mathrm{L})$ and albumin-Alexa488 $(50 \mu \mathrm{L})$ either in the early phase (MCAo+0.5h) or delayed phase (MCAo+48h) after stroke. (B\&C) Mice imaged with SP8 upright multiphoton microscope using $25 \mathrm{x}$ lense with water immersion. This region of interest exposes the somatosensory area of the cortex (specifically the barrel cortex) that is most affected by the MCAo. Imaging performed 30 min and $2 \mathrm{~h}$ after injections represented the distribution of dextran (cyan), albumin (green) and DiI- 
Lp (red) into the ipsilateral brain. The wide view images in (B) and (C) are $442 \mu \mathrm{m}$ wide and the $130 \mu \mathrm{m}$ wide ROI outlined by the white square is shown below each image. (D) At the end of the experiment mice were perfused with saline and fixed with PFA and the brains were collected and imaged with IVIS Lumina II imaging system. (E) Quantification of the fluorescent single of dextran, albumin and DiI-Lp in the brain by IVIS Lumina imaging software was performed by drawing ROI that covers the whole brain and the data expressed as total efficiency. Colour scale of epi-fluorescent signal range from $\min =1.00^{-5}$ to $\max =3.00^{-4}$ for dextran and albumin and from $\min =1.00^{-5}$ to $\max =6.00^{-4}$ for DiI-Lp. Statistical analysis of the graphs presented in E was performed using one-way analysis of variance followed by the Tukey multiple comparison test, $\mathrm{n}=3$ in each group. $\mathrm{p}$ values $<0.05$ were considered significant. 


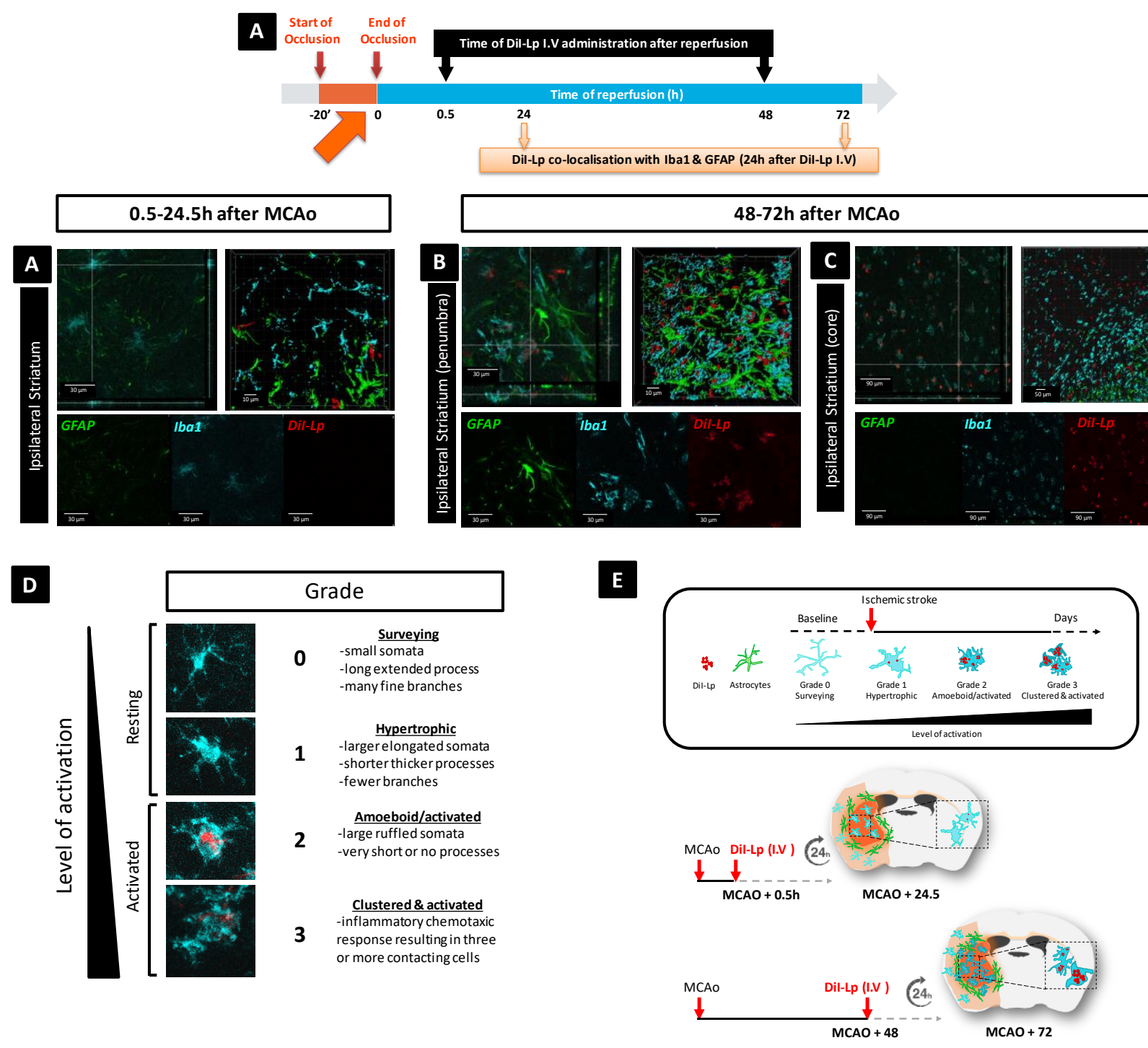

$\mathbf{F}$

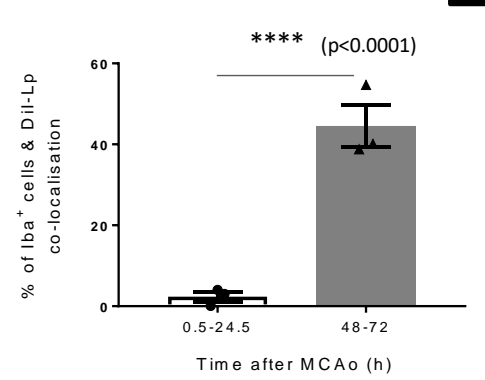

G

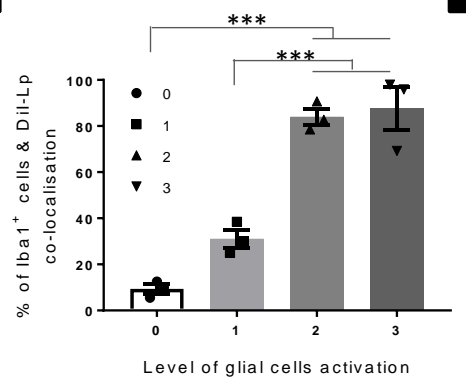

H

Figure 6: Evaluation of DiI-Lp co-localisation with microglia and astrocytes. Representative confocal images showing co-localisation of DiI-Lp with of Iba1 (activated microglial marker) and GFAP (astrocytes marker). Brain sections were analysed 24h following the injection of DiI-Lp intravenously into MCAo mice in the; (A) early phase (MCAo+0.5h) and (B\&C) delayed phase (MCAo+48h) after reperfusion. (D) Scoring system of microglial activation showing representative images of the different stages of microglial cells activation on a scale from 0-3. (E) 
Schematic summary of liposomal co-localisation with microglia and astrocytes. (F) Quantification of DiI-Lp co-localisations indicated significant uptake of DiI-Lp by microglia when injected $48 \mathrm{~h}$ after MCAo. (G) The correlations of DiI-Lp uptake by microglia with the different stages of microglial activation after MCAo. Significant uptake of liposomes by activated microglial was observed compared to minimum uptake by resting microglia $(\mathrm{H})$ No significant uptake of DiI-Lp into astrocytes was detected. Statistical analysis of $\mathrm{G}$ was performed using one-way analysis of variance followed by the Tukey multiple comparison test, $n=3$ in each group. Two-tailed unpaired student t-test used for statistical analysis of the data in F\&H (n=3-4) in each group). p values < 0.05 were considered significant. 